2006-01-01

\title{
Does a regional accent perturb speech processing?
}

\author{
Floccia, Caroline
}

http://hdl.handle.net/10026.1/9963

10.1037/0096-1523.32.5.1276

Journal of Experimental Psychology-Human Perception and Performance

American Psychological Association (APA)

All content in PEARL is protected by copyright law. Author manuscripts are made available in accordance with publisher policies. Please cite only the published version using the details provided on the item record or document. In the absence of an open licence (e.g. Creative Commons), permissions for further reuse of content should be sought from the publisher or author. 


\title{
Does a Regional Accent Perturb Speech Processing?
}

\author{
Caroline Floccia \\ University of Franche-Comté, Centre National de la Recherche \\ Scientifique-University of Provence, and \\ University of Plymouth \\ Frédérique Girard \\ University of Franche-Comté
}

\author{
Jeremy Goslin \\ University of Plymouth
}

\begin{abstract}
The processing costs involved in regional accent normalization were evaluated by measuring differences in lexical decision latencies for targets placed at the end of sentences with different French regional accents. Over a series of 6 experiments, the authors examined the time course of comprehension disruption by manipulating the duration and presentation conditions of accented speech. Taken together, the findings of these experiments indicate that regional accent normalization involves a short-term adjustment mechanism that develops as a certain amount of accented signal is available, resulting in a temporary perturbation in speech processing.
\end{abstract}

Keywords: dialects, accents, lexical access, adaptation, lexical decision

The study of accent perception and recognition offers not only a privileged insight into the phonological and prosodic representations used in speech perception but also the methods used by humans' language processing device to cope with variability. In most classical word-recognition models, interspeaker variability is largely overlooked, as it is assumed that this variability has little importance when dealing with abstract lexical representations (e.g., Lahiri \& Marslen-Wilson, 1991; McClelland \& Elman, 1986; Norris, 1994; Pallier, Colomé, \& Sebastián-Gallés, 2001). These models assume that prior to lexical matching, the acoustic signal is cleaned of all extraneous noise, resulting in a normalized representation at the prelexical level of speech processing. Although there has been considerable research on the processes behind prelexical processing and the merits of various representations (e.g., Floccia, Kolinsky, Dodane, \& Morais, 2003; Mehler, Dommergues, Frauenfelder, \& Segui, 1981; Sebastián-Gallés, Dupoux, Segui, \& Mehler, 1992; see Kolinsky, 1998, for a review), the

Caroline Floccia, Laboratory of Psychology, University of FrancheComté, Franche-Comté, France, Speech and Language Laboratory, Centre National de la Recherche Scientifique-University of Provence, Marseilles, France, and School of Psychology, University of Plymouth, Plymouth, United Kingdom; Jeremy Goslin, School of Psychology, University of Plymouth; Frédérique Girard, Laboratory of Psychology, University of Franche-Comté; Gabrielle Konopczynski, Phonetics Laboratory, University of Franche-Comté.

This research is part of a Cognitique project entitled "Ecole et Sciences Cognitives. Apprentissage des langues: Dysfonctionnements et remédiations" (2002-2004), directed by Gabrielle Konopczynski and Caroline Floccia. We thank Céline Saget and Anne Bardin for their help in data collection, Pascal Singy for his helpful comments on the description of the Swiss French accent, and Ian Dennis and Tim Perfect.

Correspondence concerning this article should be addressed to Caroline Floccia, School of Psychology, University of Plymouth, Drake Circus, Plymouth PL4 8AA, United Kingdom. E-mail: caroline.floccia@plymouth.ac.uk normalization required for the requisite processing at this level is poorly specified. It is not known exactly what kinds of variability need to be normalized at a prelexical level nor how this "irrelevant" variability could be removed. Exemplar-based models of lexical access propose a different approach, with lexical entries represented by detailed acoustic traces (Goldinger, 1996; Johnson, 1997; Klatt, 1981; Pisoni, 1997). Lexical search is then a matter of matching the incoming input to a myriad of acoustic forms stored in the lexicon, or to a quantized perceptual space, in which exemplars are encoded as weight modifications rather than through explicit storage (Johnson, 1997). This hypothesis is supported by research showing that low-level cues such as speaker identity and speaking rate are represented in long-term memory and have an impact on word-recognition tasks (Bradlow, Nygaard, \& Pisoni, 1999; Pisoni, 1997).

It is clear that the effectiveness of both methods of lexical access is reliant on an effective method of dealing with speech variability. Not only must this mechanism be able to cope with the subtle acoustic differences between similar speakers, but it must also be capable of dealing with the gross phonetic, phonological, phonotactic, and prosodic variations found among accents. Accent perception and recognition has received relatively little attention in experimental psycholinguistics compared with different types of variability, namely, speaker identity (e.g., Bradlow et al., 1999; Martin, Mullennix, Pisoni, \& Summers, 1989; McLennan \& Luce, 2005; Mullennix, Pisoni, \& Martin, 1988; see also Jusczyk \& Luce, 2002) and compressed speech (e.g., Altmann \& Young, 1993; Dupoux \& Green, 1997; Pallier, Sebastián-Gallés, Dupoux, Christophe, \& Mehler, 1998; Sebastián-Gallés, Dupoux, Costa, \& Mehler, 2000). The aim of the present study was to contribute to the debate between proponents of exemplar-based and abstract lexical representations models of word recognition, by examining the process by which a speech recognition device copes with an unfamiliar regional accent. 
Thus far, the vast majority of research on accent normalization has focused on foreign accents (Bradlow \& Bent, 2003; Clarke, 2000; Clarke \& Garrett, 2004; Gass \& Varonis, 1984; Labov, 1989; Lane, 1963; Munro \& Derwing, 1995, 1999; Schmid \& Yeni-Komshian, 1999; van Wijngaarden, 2001; Weil, 2001; Wingstedt \& Schulman, 1987), whereas regional accent adaptation has been largely overlooked, to the extent that only a single relevant empirical study has been published in this area (Evans \& Iverson, 2004). It is often acknowledged that accents can be ranked on a perceptual scale according to their acoustic distance from native speech (e.g., Clarke \& Garrett, 2004), with most foreign accents standing at the far end of the perceptual scale and regional accents standing somewhere in between. Under this assumption, the mechanisms underlying regional accent processing would simply be attenuated versions of those activated during foreign accent processing. However, there are at least two reasons to suppose that regional and foreign accents may recruit different normalization procedures. First, foreign accents embody many irrelevant variations that have no relation to the listener's maternal language, which may necessitate the activation of "offline" rescue procedures. For instance, when presented with the English accent, French native speakers would have to distinguish stress patterns, a property that is not lexically contrastive in French and has shown to cause processing difficulties in word-classification tasks (Dupoux, Pallier, Sebastián-Gallés, \& Mehler, 1997). Additionally, foreign speakers will usually be forced to adapt their own output phonology to that of a nonnative language, resulting in phonetic variations that are not known to the listener. On the other hand, although regional accents may be characterized by lexical or even syntactic differences, they mainly consist of coherent deviations in phonetic, phonological, phonotactic, and prosodic information found within the language (e.g., Wells, 1982). For example, Southern English speakers produce the word bath with an advanced, long, low, tense, unrounded vowel /a:/, whereas northern English speakers tend to use the short, lax and unrounded vowel /æ/ (Wells, 1982). Both of these phonemes are found in southern and northern English accents; therefore, in order to adapt to each other, listeners with these accents simply have to learn the differences in the lexical distribution of these two vowels. On the contrary, the vowel /æ/ does not form part of the phonemic inventory of Parisian French speakers (see Tranel, 1987). Also, although their inventory does contain /a/, the long version /a:/ also exists, but this length distinction is not lexically contrastive. As a consequence, when a Parisian French speaker produces the vowel of the word bath, the speaker can target three possible realizations: /æ/, /a/, and /a:/. However, the first of these vowels will not be familiar to the speaker, and the second may not be familiar to native English speakers; as in Received Pronunciation English, this vowel only exists in its longer form, $(/ \mathrm{a}: /)$. Therefore, one can see that the variability in the speech signal resulting from the foreign Parisian French accent is likely to require more complex normalization mechanisms than those involved in differentiating differences between the lexical distribution of /æ/ and /a:/ between the north and the south of England.

With both qualitative and quantitative differences between regional and foreign accents, it is clear that a thorough understanding of underlying normalization processes will require further empirical research linking adaptation to both types of accents. To this end, we present a series of six experiments designed to reveal the initial cost caused by exposure to a regional accent in continuous speech in comparison with that involved with foreign accents. This study will be presented in light of the available literature on accent normalization and the theoretical predictions of two models of lexical access that make specific claims for accent processing (Johnson, 1997; Norris, McQueen, \& Cutler, 2003).

\section{Comprehension Disruption Resulting From a Foreign Accent}

As with any other normalization mechanism, accent adaptation should be typified by two distinct phases: an initial period, in which comprehension is disrupted, followed by an adaptation phase leading to the total or partial recovery of baseline comprehension. Language processing impairments due to foreign accents have been noted in a number of studies, and they have been found to affect both syntactic and semantic processing. In a study by Munro and Derwing (1995), both comprehension and truth judgment tasks revealed that foreign accents-in this case, Chinese speakers producing English sentences-delayed judgments by an average of $30 \mathrm{~ms}$ compared with native accents. Similarly, Schmid and Yeni-Komshian (1999) presented English listeners with sentences containing intentional pronunciation errors produced by native and nonnative speakers. As in the previous study, they found that their participants were significantly better at detecting the errors when the sentences were produced by native speakers. This led to the conclusion that foreign accent processing comes at a cost (also see Lane, 1963; van Wijngaarden, 2001), requiring the intervention of top-down mechanisms similar to those used for phonemic restoration.

\section{Adaptation to a Foreign Accent}

One of the clearest examples of foreign accent adaptation was shown in a seminal study by Gass and Varonis (1984), in which participants were asked to transcribe sentences produced by a nonnative speaker. They found that transcription accuracy improved if participants were first exposed to a story also read in the same nonnative accent, suggesting that prolonged exposure to an accent could reduce associated processing costs (also see Bradlow \& Bent, 2003; Clarke, 2000; Weil, 2001; Wingstedt \& Schulman, 1987). This study was then extended by Clarke and Garrett (2004), who went on to investigate how much prior exposure was necessary for full foreign accent adaptation to take place. In this study, three groups of native English speakers were tested in a crossmodal matching task, which tested the time taken to match visual probes with the final word of test sentences produced with either a native accent or a Spanish accent. Each group was exposed to 16 sentences, each having duration of $2 \mathrm{~s}$. In the first group, the 12 initial sentences were produced in participants' native accent, followed by 4 in a Spanish accent. In the second group, all of the sentences were produced with a Spanish accent, and they were produced in the third group by a native accent. Clarke and Garrett found that listeners initially took on average 100-150 ms longer to identify the target words when the target sentences were produced with the Spanish accent, rather than the native accent, revealing a strong disruption effect of the accent. They also found that adaptation to a foreign accent, shown by a resumption of baseline reaction times (RTs), required less than $1 \mathrm{~min}$ of prior exposure to 
the accent and that, under some circumstances, this could occur after prior exposure to only 2-4 sentences. These findings confirmed the existence of a two-stage normalization process, with initial disruption of comprehension followed by a rapid adaptation; however, these findings do not shed any light on the origins of these two mechanisms (lexical vs. prelexical, bottom up or top down).

\section{Adaptation to a Regional Accent}

To our knowledge, the only relevant empirical study on the topic of regional accent adaptation is that of Evans and Iverson (2004), who examined how vowel perception is modified to accommodate regional accent differences. Listeners from either the north or south of England were asked to rate synthesized vowels embedded in carrier words according to their match with natural equivalents. Prior to this step, each of the participants was presented with a 2-min story spoken with either a familiar or unfamiliar accent to habituate them to a particular accent. They found that the listeners adjusted their responses in the vowel-categorization task according to the accent used in habituation and that their listeners differed from other descriptions of how listeners perceive foreign-accented speech. That is, whereas foreign-accented phonemes tend to be assimilated into native categories (e.g., Best, McRoberts, \& Goodell, 2001; Flege, 1995), listeners in this task were able to adjust to regional phonemic variations and change their responses accordingly. This finding suggests that a regional accent can lead to modifications of the phonological representations used for analyzing the incoming speech signal, rather than to post hoc shifts in phonemic categorization found with foreign accents. This study further reinforces the idea that it is necessary to distinguish between regional and foreign accent normalization.

\section{Theoretical Accounts of Accent Normalization}

A number of speech processing models have direct bearing on accent adaptation, although none have been specifically designed to account for these types of speech variations. For instance, an exemplar-based model of speech perception developed by Johnson (1997) does not include any speaker normalization processes per se; however, the auditory properties that distinguish speakers, such as accent, are retained in the exemplars. In this model, accent variations are compensated by the variability inherent in the remembered exemplars; therefore, some effects of accent familiarity would emerge naturally without the application of a normalization mechanism. A foreign accent would disrupt normal comprehension because it violates the usual frame of reference. However, continued exposure to a foreign accent would result in a progressive shift in the weights used to represent the speech signal leading to adaptation and a return to baseline speech processing performance.

Even if abstract entries are used for lexical storage, accentrelated variability can still disrupt normal comprehension because of inadequate prelexical processing. When discussing the merits of lexical feedback to assist online perception or perceptual learning, Norris et al. (2003) claimed that, over time, information available from the lexical level of analysis can lead listeners to retune their prelexical categories. They postulated that the interpretation of prelexical information can be altered by providing top-down information from the lexicon in the form of a training signal. According to this model, an unfamiliar accent would cause initial disruption due to inaccurate prelexical processing. When the listener eventually establishes the identity of the words, lexicon can begin to instruct the prelexical processing levels to interpret mismatched phonemes or accent patterns to fit the unfamiliar accent.

This proposal complements those made by Dupoux and Green (1997) after an investigation of humans' ability to rapidly adapt to compressed speech. They proposed that this type of adaptation results from the conjugate action of two mechanisms, a short-term adjustment to local parameters and a long-term learning process that encodes phonological and lexical information from this new speech style. By suggesting that these same mechanisms are used to adapt to either regional or foreign accents, Dupoux and Green proposed that, parallel to the accent-normalization process, listeners must be able to extract information about the encountered accent and store it in long-term memory (see Clarke \& Garrett, 2004, for a similar proposition). Although this accent template bank has sociolinguistic application, allowing researchers to identify a given accent, it can also be used to accelerate and optimize online accent-normalization mechanisms.

As we have seen, the literature is rich with studies showing impaired speech processing due to presentation of foreign accents, with broad theoretical accounts of possible adaptation mechanisms. However, further empirical data are clearly required before researchers can reach an understanding about the underlying normalization processes involved in accent adaptation, especially in regional accent perception. In this study, we investigated the perceptual cost involved in processing these accents, allowing us to discuss the mechanisms used to adapt within the coherent phonological and prosodic variation of the maternal language. In each of the six experiments, lexical decision tasks were used to compare differences in detection latencies for target wordspseudowords placed at the end of sentences produced by speakers with different regional accents. By measuring the word identification cost due to an unfamiliar accent over continuous speech, we found that accent-related variations are not simply limited to phonemic or phonotactic differences but extend to prosodic differences (for a similar methodological choice, see also Bradlow \& Bent, 2003; Clarke \& Garrett, 2004; Munro \& Derwing, 1999; Schmid \& Yeni-Komshian, 1999). Listeners were presented with all of the linguistic cues characterizing a nonfamiliar accent, offering an ecologically valid basis for the testing of processes involved in accent normalization. Naturally, this methodological approach is not without its own concerns, as it does not allow for the precise control of all aspects of accent-related variability. However, in an effort to alleviate potential methodological concerns over the locus of effects attributed to accent, we examined the perception of five regional French accents (Franche-Comte, Aix-en-Provence, Toulouse, Swiss French from Fribourg, and Parisian) in two French populations (the Franche-Comté region in Experiments 1-5 and the Toulouse region in Experiment 6), with examples of each accent being produced by multiple speakers (see also Munro \& Derwing, 1999; Schmid \& Yeni-Komshian, 1999), to generalize our findings across speakers, accents, and the listeners' regional origins. 


\section{Experiment 1}

This aim of this experiment was to ascertain the existence of a speech processing cost due to unfamiliar regional accents. This hypothetical processing cost was examined by asking participants to make a lexical decision on the final word of sentences produced by speakers with regional French accents that are native, familiar, and unfamiliar to the participants. Foreign-accent processing costs of between 100 and $150 \mathrm{~ms}$ were observed by Clarke and Garrett (2004), using a cross-modal matching task on sentence-ending words. However, according to their proposition of accent ranking (regional accents being perceptually less distant from the familiar input than foreign accents), full adaptation should occur more rapidly in our experiment, and the word identification cost associated with an unfamiliar regional accent should be smaller in size.

\section{Method}

Participants. Twenty participants (including 6 men) with an average age of 23 years 11 months were tested in this experiment. In this and all other experiments apart from Experiment 6, participants were monolingual French students at the University of Franche-Comté with no known hearing defects. The regional linguistic origin of the participants was ascertained by questionnaire, ensuring that all of the participants were local to the Franche-Comté region. Moreover, any participant who had been absent from the region for over a year or who had parents who were not native to the region was excluded from the experiment. The same recruitment procedure was used in all experiments in the study, except for Experiment 6.

Regional accents in French. Phonological and phonetic analyses of French suggest a broad perceptual boundary between northern, including the so-called "standard" Parisian accent conveyed by the media, and southern accents (Carton, Rossi, Autesserre, \& Léon, 1983; Hintze, Pooley, \& Judge, 2001). This also corresponds to the boundary between the dialects of Oil in the north, east, west, and south-central regions and the dialects of Oc, which cover the south part of France. A third family is identified as the Franco-Provençal dialects, which include the region between Grenoble, Lyons, and Geneva as well as the Suisse Romande part of Switzerland and the Aoste Valley in Italy (Battye, Hintze, \& Rowlett, 2000; Rash, 2002; Singy, 2002). Further diversity is also evident within each of these regions and is now being evaluated as part of a database collected under the framework of the international Francophone project, "Contemporary French Phonology" (Durand, Laks, \& Lyche, 2002). In our experiments, we examined the effect of French regional accent variations by contrasting processing costs for three types of accent we have labeled as home, familiar, and unfamiliar.

The first of these, the home accent, refers to the regional accent spoken by all of the participants of this study (apart from those in Experiment 6). This accent is local to the Franche-Comté region of northeastern France and is perceptually (and geographically) close to the French-speaking Swiss accent, although it belongs to a different dialect family (Oil vs. Franco-Provençal). The second accent was categorized as familiar, as it refers to the Parisian accent, familiar to all residents of France because it is the accent predominantly used in the French mass media (see Tranel, 1987, for a description). The Franche-Comté home accent is typified by a number of differences to the Parisian accent, including the closure and lengthening of vowels in closed syllables (that normally should be open and short: neige [snow] produced as /ne:3/ instead of /nع3/) and the opening of vowels when they are in the word-final position (normally should be close: pot [tin] produced as /po/ instead of /po/). There is also a tendency for $/ \mathrm{r} /$ and some voiced occlusives to be unvoiced (encore [again] produced as /ãkoR/instead of /ãkoR/). Also, although Parisian speakers would tend to lengthen the last syllable of a word, speakers from the
Franche-Comté region will use stress in diverse syllabic positions (maison [house] produced with a first long syllable and a second short, instead of the reverse pattern; Konopczynski, 1979; Rittaud-Hutinet, 2001).

Our unfamiliar accents comprised two very similar accents from the south of France, each very different from the accents found in any other region of France. The first of these, from Aix-en-Provence (used in Experiment 1), is typified by the consistent closure of /e/ in open syllables (avais [had] produced as /ave/ instead of /ave/) and by representing $a$ with a front vowel instead of a back vowel used in other French accents. Other features include the production of nasal vowels with nasal consonant

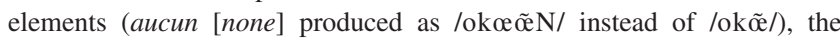
simplification of intervocalic consonant clusters (/gz/ becomes /z/), and the devoicing of $/ \mathrm{r} /$ when it appears in the word-final position, or rounding in other positions. In addition, the consistent group stress found on the final syllable of utterances in other accents will move if the final word ends in a schwa (in le ver de terre [the worm], the accent is carried by the two syllables of terre, /terə/). Our second unfamiliar accent, from Toulouse in the southeast of France (used in Experiments 2-6), is very similar to that of Aix-en-Provence (they also happen to be geographically close, even if this is not a sufficient condition). Only minor features differentiate these accents, such as the closure of vowels (cordes [strings], produced as /koRd/ instead of $/ \mathrm{koRd} /$ ), systematic production of schwa (uniquement [only], produced as /ynikəmã/ instead of /ynikmã/; although it is not produced in front of a vowel), and consonant germination in certain cases (dans le circuit [in the circuit] produced /dãlləsirkui/ instead of /dãləsirkui/; see Carton et al., 1983).

The Swiss French accent (used in Experiment 6) belongs to the FrancoProvençal dialect set (Matthey, 2003; Rash, 2002). Even though this accent varies depending on the region or canton, it is generally characterized by its resistance to oxytonie. That is, contrary to that of the inner French accents, French-speaking Swiss do not accentuate the last syllable of a word or group but any other syllable (Knecht \& Rubattel, 1984; Matthey, 2003). Another characteristic of this accent is the relatively slow speaking rate, when compared with other French dialects. This feature has never been investigated seriously but forms part of the intuitions of Swiss linguists and speakers (Singy, 2004). Additional distinctive features of this accent include the use of final vowel length to distinguish gender on words such as amie versus ami (girlfriend vs. boyfriend; /ami:/vs. /ami/) and a tendency to produce the phonemes $/ \mathrm{J} /$ and $/ 3 /$ as $/ \mathrm{t} \int /$ and $/ \mathrm{t} 3 /$, respectively). Recordings of all of the accents used in this study can be obtained from Caroline Floccia.

Stimuli. Target items consisted of 19 high-frequency disyllabic words (mean frequency in Lexique, designed by New, Pallier, Ferrand, \& Matos, 2001: 66.4 , from 5 to 399) and 10 pseudowords. Each of these words was placed at the end of 12 carrier sentences, 4 for each of the accents under analysis. For example, in the carrier sentence Anne n'a jamais vu de mouton (Ann has never seen any sheep), participants were asked to make a lexical decision on the target word mouton (sheep). Carrier sentences were formed from three different syntactic frames, comprising between 6 and 14 syllables. In addition, each sentence was constructed such that the final word of the sentence would remain ambiguous until it was spoken (as ascertained by a pilot completion test). Sentences produced for the three regional accents were split between two female speakers native to their respective regions. Multiple speakers were used for each region to ascertain whether any accent effects were general to the regional characteristics of speech rather than to the idiosyncrasies of a particular speaker. This organization of stimuli resulted in a total of 348 different sentences, with each speaker producing a subset of 58 sentences. A list of the target words and pseudowords used in this experiment can be seen in the Appendix.

No attempt was made to control for the particular phonetic or phonological features characterizing each accent in the target stimuli or in the carrying sentences. It was supposed (and subsequently validated) that any chosen sentence would carry sufficient information about the speaker's accent. 
Procedure. The experiment was controlled using the software EXPE (Pallier, Dupoux, \& Jeannin, 1997) with sentences presented in two equalsized blocks (separated with a pause) in random order with an interstimulus interval of $6 \mathrm{~s}$. Lexical decisions were indicated in a go/no-go task, with participants pressing a button with their favored hand if the target, being the final word-pseudoword of the sentence, was lexical. RTs were measured from the onset of the target. A small number of training sentences were presented before the main experimental block, although none of these sentences were subsequently used in the main experiment.

\section{Results}

Out of the 4,560 required lexical decisions, 9 were missing, 24 were anticipatory (decision made before the target word was presented), and 75 were rejected as being too slow (RT above 2.5 times the standard deviation of each participant), leaving 4,452 lexical response times $(2.36 \%$ error rate). For the 2,400 pseudowords, participants responded with 52 false alarms $(2.2 \%$ error rate).

Lexical response times were examined using an analysis of variance (ANOVA), with two within-participant variables: accent (three modalities: home, familiar, and unfamiliar) and speaker (two speakers per accent). The main effect of accent was found to be significant by participant $\left(F_{1}\right)$ and item $\left(F_{2}\right), F_{1}(2,38)=17.29$, $p<.001$, and $F_{2}(2,36)=8.21, p=.0012$, because of longer RTs in detecting words with unfamiliar accent $(743 \mathrm{~ms})$ than those with familiar (711 ms) and home (709 ms) accents. Further analyses showed that although the difference between familiar and home accents was not significant, $F_{1}(1,19)<1$, and $F_{2}(1,18)<1$, there was a significant difference between the unfamiliar accent and both the home, $F_{1}(1,19)=33.69, p<.001$, and $F_{2}(1,18)=$ $18.74, p<.001$, and familiar, $F_{1}(1,19)=26.80, p<.001$, and $F_{2}(1,18)=6.95, p=.017$, accents. Finally, speaker effects were not found to be significant for any of the familiar, $F_{1}(1,19)<1$, and $F_{2}(1,18)<1$, or home, $F_{1}(1,19)=3.86, p=.064$, and $F_{2}(1$, $18)<1$, accents, and was only found to be marginally significant in the unfamiliar accent, $F_{1}(1,19)=4.42, p=.049$, and $F_{2}(1$, $18)=1.81, n s$.

These results show that there is a significant increase in lexical RTs associated with the processing of unfamiliar accents. Furthermore, as there was no robust difference in the RTs between the two speakers of each of the accents, we can assume that they were each representative of their linguistic origins. This indicates that the observed between-accents differences were due to differences in accent, rather than to individual idiosyncrasies of our speakers; therefore, we did not examine interspeaker differences in further analyses.

Although the unfamiliar accent gave rise to longer detection latencies, there remains the possibility that this effect could be due to basic differences in stimuli across accents, rather than to a reflection of increased processing costs. That is, speakers from the unfamiliar accent region could have spoken at a different rate than those of the other regions. The unfamiliar southern French accent is characterized, among other things, by restoration of the schwa that could lead to slower speaking rates than the other, northern, accents. Rate normalization or compressed speech studies have shown that rate changes can produce delays in word identification (see Dupoux \& Green, 1997). Also it is well known that increasing the length of an auditory token also increases identification responses. ${ }^{1}$
In order to examine the relation among RTs, target word length, and sentence length, we computed separate regression equations for each participant and for each accent. Each equation involved regression from the 76 observations from each participant to the two predictor variables, namely, target word length and sentence duration (up to the beginning of the target word). To test whether each regression coefficient differed reliably from zero, we ran single group $t$ tests for each variable coefficient set. This analysis follows the recommendation of Lorch and Myers (1990) for regression analyses of repeated measures data.

As can be seen in Table 1, the coefficients of regression relating RTs and target word length were significantly positive, with longer words increasing RTs. However, although unfamiliar accent words were found to be significantly longer than home accent words, $F(1$, $75)=24.26, p<.001$, their duration was similar to that of the familiar accent words, $F(1,75)<1$. This indicates that target word durations are unlikely to be the cause for the differences in RT latencies between accents.

A similar comparison of target RT and sentence duration shows that utterance duration, also shown in Table 1, was significantly longer for the unfamiliar accent. However, an examination of RT and sentence duration within each accent revealed significant negative regression coefficients between the two factors in home and unfamiliar accents. Therefore, it appears that, within each of the accents, there is an inverse relationship between sentence length and RTs, possibly because of a classical signal-go effect or also an increase in word predictability with longer sentences. In any case, these within-accent effects do not explain our betweenaccents differences, as the sentences produced in the unfamiliar accent have both the longest duration and RT.

\section{Discussion}

The main result of this first experiment is that an unfamiliar regional accent elicits a $30-\mathrm{ms}$ delay in word identification in continuous speech. This can be compared with a related study by Clarke and Garrett (2004), who reported a 100- to 150-ms delay in word identification with a foreign accent. This apparent difference in processing delays between regional and foreign accents would appear to give credence to the claim of Clarke and Garrett that postulates that accents can be ranked on a perceptual scale as a function of their distance from the native home accent. Regional accents would stand between the reference accent and the foreign accents and would thus trigger fewer mismatch signals when first presented to the listeners. However, a more pragmatic reason for the difference in the magnitude of the delay could also stem from Clarke and Garrett's use of a cross-modal matching task, which would tend to consume more resources than our lexical decision task, resulting in increased RT delays. However, before entering into this discussion too deeply, we must further investigate to confirm whether the effects we have found in this first exploratory experiment were due to the unfamiliarity of an accent in continuous speech and not to other confounding variables.

\footnotetext{
${ }^{1}$ We avoid mentioning correlations between RTs and position of the unicity point, because for all target words but one, the phonological unicity point falls on the last phoneme (for melon, the unicity point is on the third phoneme, /1/).
} 
Table 1

Mean Reaction Times, Target Word, and Sentence Duration (up to the Beginning of the Target Word) in Experiment 1 (in Milliseconds)

\begin{tabular}{|c|c|c|c|c|c|c|c|c|c|}
\hline \multirow[b]{2}{*}{ Accent } & \multirow{2}{*}{$\begin{array}{c}\text { Target word } \\
\text { duration }\end{array}$} & \multicolumn{2}{|c|}{$B$} & \multirow[b]{2}{*}{$t(20)^{\mathrm{a}}$} & \multirow{2}{*}{$\begin{array}{l}\text { Sentence } \\
\text { duration }\end{array}$} & \multicolumn{2}{|c|}{$B$} & \multirow[b]{2}{*}{$t(20)^{\mathrm{b}}$} & \multirow[b]{2}{*}{$\begin{array}{c}\text { Mean } \\
\text { RT }\end{array}$} \\
\hline & & $M$ & $S D$ & & & $M$ & $S D$ & & \\
\hline Home & 430 & 0.68 & 0.23 & $13.09 *$ & 1,364 & -0.03 & 0.02 & $-4.78 *$ & 711 \\
\hline Familiar & 466 & 0.68 & 0.36 & $8.61 *$ & 1,386 & -0.02 & 0.04 & -1.59 & 709 \\
\hline Unfamiliar & 472 & 0.64 & 0.25 & $11.31 *$ & 1,670 & -0.04 & 0.05 & $-3.57^{*}$ & 743 \\
\hline
\end{tabular}

Note. $\quad \mathrm{RT}=$ reaction time.

${ }^{a}$ Mean coefficients and associated $t \mathrm{~s}$ for regression of reaction times on target word duration. ${ }^{\mathrm{b}}$ Mean coefficients and associated $t$ s for regression of reaction times on sentence duration.

$* p<.05$.

The first possible confound we wish to address is the possibility that the disparity in RT between familiar and unfamiliar accents could be due to differences in the production of the target words. That is, rather than revealing word identification disruption due to the preceding sentence, the difference in RT observed in Experiment 1 could be due to the accent carried by the test item itself. In the second experiment, we have attempted to differentiate between these factors by testing lexical decision on isolated words versus words embedded in sentences.

\section{Experiment 2}

In this experiment, we examined whether the processing cost due to an unfamiliar accent found in Experiment 1 is evident in the presentation of isolated words. Participants were asked to make a lexical decision on target words produced in either their home accent or in an unfamiliar accent (from the Toulouse region). The target words were presented both in isolation and at the end of a carrier sentence (as in Experiment 1).

\section{Method}

Participants. Thirty-three participants (including 4 men) with an average age of 23 were tested in this experiment. Three additional participants were tested but rejected: 1 whose RTs were too slow (above $800 \mathrm{~ms}$ ), another for failing to understand the task, and another for missing too many trials (more than 50\%).

Stimuli. Target items consisted of 10 high-frequency disyllabic words (mean frequency from Lexique: 35.4, from 5 to 104) and six pseudowords, each of which started with an unvoiced plosive to facilitate extraction. As in the previous experiment, two female speakers were used to produce sentences for each of the accents (home accent and unfamiliar accent from Toulouse). Two sets of 64 sentences were used (comprising 12 to 14 syllables each), the first set forming a block of stimuli (sentence block) in which the targets were presented at the end of their respective carrier sentence. For the second set of sentences, each of the target words was extracted for presentation in isolation. Each of these extracted words was presented twice in a randomized block of 128 isolated words-pseudowords (isolated target block). A list of all of the target words and pseudowords used in this experiment can be seen in the Appendix. ${ }^{2}$

Procedure. The procedure was the same as in Experiment 1, diverging only in the software used to control the experiment, changed to EPRIME (Schneider, Eschman, \& Zuccolotto, 2002), and the length of the interstimulus interval, which was now 3 seconds. Of the 33 participants tested in this experiment, 8 were randomly assigned to the word-sentence group. In this group, the participants were initially presented with the isolated target block and then the sentence block; the remaining participants were assigned to the sentence-word group, where the block order was reversed.

\section{Results}

Out of the 3,960 required lexical decisions, 38 were missing, 3 were anticipatory, and 99 were rejected as being too slow (RT above 2.5 times the standard deviation of each participant), leaving 3,820 valid lexical response times ( $3.5 \%$ error rate). For the 2,376 pseudowords, participants responded with 106 false alarms $(4.5 \%$ error rate).

ANOVAs of the RT responses of our participants were performed with one between-participants variable, presentation order (word-sentence or sentence-word), and three within-participant variables, accent (home or unfamiliar), target type (isolated or sentence carrier), and speaker (two per accent). Examining the potential effect of presentation order, we found that there was no significant interaction between this factor and accent, $F_{1}(1,31)<$ 1 , and $F_{2}(1,18)<1$, nor speaker, $F_{1}(1,31)<1$, and $F_{2}(1,18)<$ 1. However a significant interaction was found with target type, $F_{1}(2,62)=10.52, p<.001$, and $F_{2}(2,36)=28.49, p<.001$, revealing a familiarization effect, with responses tending to be faster in the second block than in the first. An effect of speaker was found with the home accent, $F_{1}(1,31)=30.8, p<.001$, and $F_{2}(1$, $18)=10.22, p=.005$, but not with the unfamiliar accent, $F_{1}(1$, $31)=2.23$, and $F_{2}(1,18)<1$.

A main effect of accent was observed, $F_{1}(1,31)=7.98, p=$ .008 , and $F_{2}(1,18)=2.09, p=.17$, showing that participants were faster with the familiar accent than with the unfamiliar one (587.7 ms vs. $598.1 \mathrm{~ms}$, respectively). Although there was no significant interaction found between the factors of accent and target type, $F_{1}(2,62)=1.88$, and $F_{2}(2,36)=1.26$, an effect of accent was observed when targets were preceded by a sentence, $F_{1}(1,31)=$ $6.65, p=.015$, and $F_{2}(1,18)=1.75$, but not when they were presented in isolation, $F_{1}(1,31)<1$, and $F_{2}(1,18)<1$ (see Figure 1).

\footnotetext{
${ }^{2}$ Experiments 2-6 used a different set of stimuli than Experiment 1 because they were part of a project involving testing of young children. Stimuli were selected as a function of their high frequency in Lexique (New et al., 2001) but also as a function of their familiarity with that population.
} 


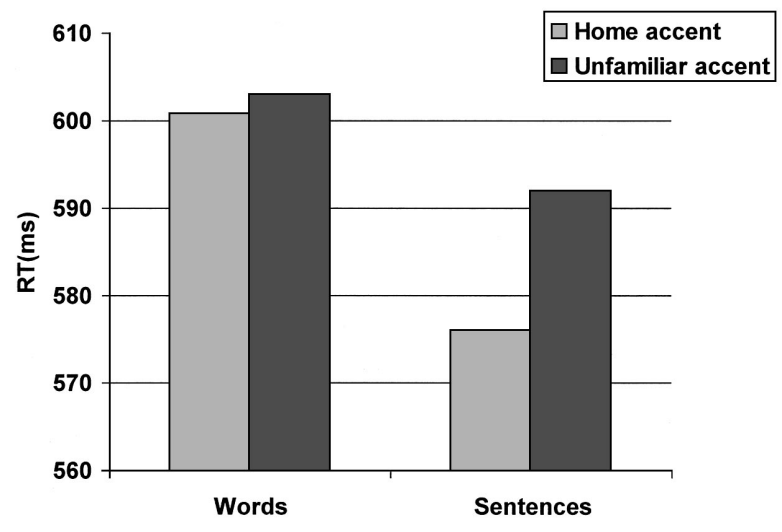

Figure 1. Mean reaction times (RTs) as a function of target stimulus context and accent familiarity in Experiment 2.

Contrary to the previous experiment, no correlation was found between sentence duration and lexical decision times in the sentence block, as revealed by the distribution of individual regression coefficients (mean $B=0.01$ ), $t(32)=1.22$. This finding showed that equalizing the sentence duration in this block resulted in the disappearance of the signal-go effect found in the previous experiment. However, regression of RTs on target word length resulted in significantly positive coefficients, both in the isolated word block (mean $B=0.09$ ), $t(32)=5.88, p<.001$, and in the sentence block (mean $B=0.31$ ), $t(32)=8.98, p<.001$. As can be seen in Table 2, the duration of the targets produced by the home accent speakers was shorter than those of the unfamiliar accent. However, it is unlikely that these differences could account for the accent effect, as this was found only for stimuli in the sentence block. If stimulus duration was the cause of the effect of accent, we would expect to see the same effect in both blocks, as there are similar durational differences for the targets across blocks.

\section{Discussion}

The main aim of this experiment was to evaluate the locus of the accent effect seen in Experiment 1 (e.g., to ascertain whether the processing cost of unfamiliar accents seen in that experiment was due to accent differences in the target word or to differences in the carrier sentences). In this experiment, a main effect of accent familiarity was found, which was not significantly weaker for isolated target words than for those preceded by a carrier sentence. This suggests that the word identification cost associated with unfamiliar accents observed in Experiment 1 could have been due to the accent on the target, rather than to a normalization process passed down along the carrier sentence.

However, the isolated words used in Experiment 2 were extracted from a carrier sentence and, as such, carry suprasegmental information not normally found in words uttered in isolation. This could explain the lack of a significant difference between isolated words and those found in carrier sentences, whereas, as can be seen in Figure 1, there is a tendency for a stronger effect of accent when carrier sentences are used. In light of these inconclusive results, the next experiment was designed to investigate the significance of signal duration on accent adaptation by examining the effect of unfamiliar accents as a function of duration of the whole utterance.

\section{Experiment 3}

The main aim of this experiment was to examine whether the accent-related word identification cost is sensitive to signal length in continuous speech. By varying the length of carrier sentences, we aimed to ascertain how much signal is required to trigger accent detection mechanisms. Contrary to the previous experiment, which compared isolated words and sentences, Experiment 3 was designed to contrast full-formed sentences of different lengths (short: 7-9 syllables; middle length: 12-14 syllables; and long: 17-19 syllables), therefore providing participants with the complete set of phonological and suprasegmental accent-related information. One prediction for this experiment was that listeners may need to gather a certain amount of unfamiliar accent evidence before triggering the accent normalization process, after which a constant word identification cost should be observed across all lengths of carrier sentence. Alternatively, it is possible that disruption decreases as more signal is made available, suggesting that subsequent adaptation is taking place.

\section{Method}

Participants. Thirty-five participants (including 6 men) with an average age of 21 years 5 months were tested in this experiment. Three additional participants were tested but rejected: 1 whose RTs were too slow (above $800 \mathrm{~ms}$ ) and the others for missing too many trials (more than 50\%).

Stimuli. In this experiment, both the target words and pseudowords used in Experiment 2 were used in the generation of three blocks of sentences, each of differing length. As in the previous experiments, each block consisted of 64 different sentences presented in random order, one for each of the targets produced by four speakers (two speakers each for the home and unfamiliar Toulouse accent). The three blocks consisted of short (7-9 syllables), medium (12-14 syllables; this block is identical to that used in the previous experiment), and long (17-19 syllables) sentences.

Procedure. After a training session, all participants were initially presented with the medium block, with the order of presentation for the short and long blocks counterbalanced across participants.

\section{Results}

Out of the 4,200 required lexical decisions, 21 were missing and 108 were rejected as being too slow (RT above 2.5 times the

Table 2

Mean Word Duration, Sentence Duration (up to the Beginning of the Target Word), and Reaction Times in Experiment 2 (in Milliseconds)

\begin{tabular}{|c|c|c|c|c|c|}
\hline \multirow[b]{2}{*}{ Accent } & \multicolumn{2}{|c|}{ Isolated words } & \multicolumn{3}{|c|}{ Sentences } \\
\hline & Duration & $\begin{array}{c}\text { Mean } \\
\text { RT }\end{array}$ & $\begin{array}{l}\text { Final word } \\
\text { duration }\end{array}$ & $\begin{array}{l}\text { Sentence } \\
\text { duration }\end{array}$ & $\begin{array}{c}\text { Mean } \\
\text { RT }\end{array}$ \\
\hline \multicolumn{6}{|l|}{ Familiar } \\
\hline Speaker 1 & 532 & 589 & 477 & 1,855 & 569 \\
\hline Speaker 2 & 542 & 613 & 518 & 1,564 & 584 \\
\hline \multicolumn{6}{|l|}{ Unfamiliar } \\
\hline Speaker 1 & 584 & 608 & 567 & 1,641 & 593 \\
\hline Speaker 2 & 580 & 599 & 526 & 1,681 & 591 \\
\hline
\end{tabular}

Note. $\quad \mathrm{RT}=$ reaction time. 
standard deviation of each participant), leaving 4,071 lexical response times (3.0\% error rate). For the 2,520 pseudowords, participants responded with 70 false alarms ( $2.8 \%$ error rate).

ANOVAs of the RT responses of our participants were made with one between-participants variable, presentation order (medium-short-long or medium-long-short), and three withinparticipant variables, accent (home or unfamiliar), carrier sentence length (short, medium, and long), and speaker (two per accent). Examining the potential effect of presentation order, we found that there was no significant interaction between this factor and accent, $F_{1}(1,33)=3.04, p=.09$, and $F_{2}(1,18)=1.08$. However, a significant interaction was found with sentence length, $F_{1}(2,66)=$ $4.54, p=.014$, and $F_{2}(2,36)=6.18, p=.005$, revealing a familiarization effect, with responses tending to be slower in the first block of medium-length sentences than the following blocks of short and long sentences, $F_{1}(1,33)=10.53, p=.003$, and $F_{2}(1$, $18)=48.86, p<.001$. An effect of speaker was found with the unfamiliar accent, $F_{1}(1,33)=38.8, p<.001$, and $F_{2}(1,18)=$ $11.1, p=.004$, but not with the home accent, $F_{1}(1,33)<1$, and $F_{2}(1,18)<1$.

The factor of regional accent was also found to have a significant effect in this experiment, $F_{1}(1,33)=18.8, p<.001$, and $F_{2}(1,18)=8.41, p=.0096$, with lexical decisions significantly faster for sentences produced with a home accent than the unfamiliar accent (558.9 ms vs. $573.6 \mathrm{~ms}$, respectively). The effect of regional accent was also found to interact with sentence length, $F_{1}(2,66)=7.10, p=.0016$, and $F_{2}(2,36)=2.99, p=.06$, even when the medium-length sentences were removed (because of the possible block order effect) and only short and long sentences were compared, $F_{1}(1,33)=11.29, p=.002$, and $F_{2}(1,18)=5.77, p=$ .027 .

As can be seen in Figure 2, the processing cost associated with unfamiliar accents increases along with the length of the sentence. In short sentences, the difference between accents is not significant, $F_{1}(1,33)<1$, and $F_{2}(1,18)<1$, however, this rises to significance with medium sentences, $F_{1}(1,33)=8.86, p=.0054$, and $F_{2}(1,18)=1.86$, and increases still further with long sentences, $F_{1}(1,33)=22.94, p<.001$, and $F_{2}(1,18)=11.93, p=$ .0028 .

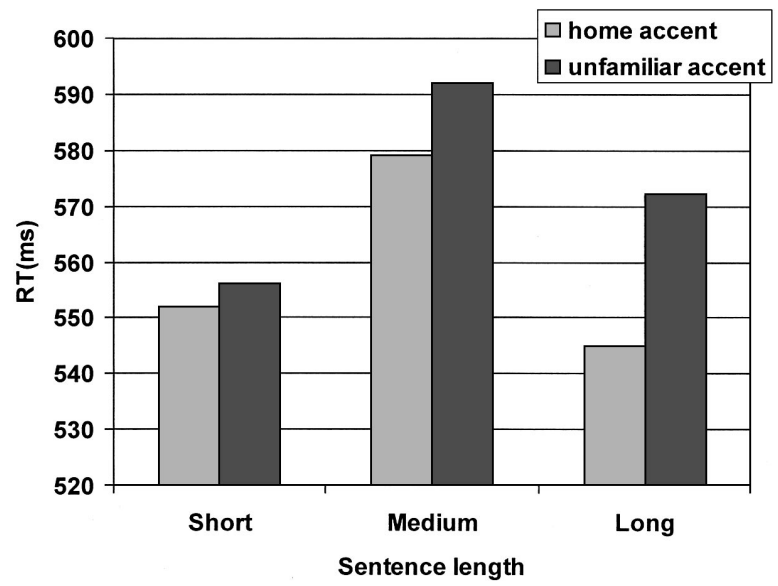

Figure 2. Mean reaction times (RTs) as a function of sentence length and accent familiarity in Experiment 3.
Regression analyses of RTs revealed that both sentence duration (mean $B=0.011), t(34)=2.87, p=.007$, and target word length (mean $B=0.25), t(34)=8.93, p=.000$, were significantly correlated with RTs, with longer words eliciting slower RTs. An examination of mean RTs for each of the speakers, shown in Table 3 , reveals that one of the unfamiliar accented speakers elicited considerably longer RTs than the other speakers. However, the duration of target words produced by this speaker was not significantly longer than those produced by the other speaker from the unfamiliar accent group, $F(1,9)=1.02$. Therefore, it is unlikely that the slow RTs with this speaker can be attributed to target duration.

\section{Discussion}

The findings of this experiment reveal that the word identification costs associated with the processing of unfamiliar accents increase with signal length, indicating that the process of regional accent adaptation gradually impairs comprehension as information on the accent is gathered from the speech signal. At first glance, these results contrast with those of Experiment 2, in which we failed to obtain a clear interaction between target type (isolated words vs. sentences) and accent familiarity. However, some light might be shed on these inconsistencies if we compare the effects of accent seen with medium sentences with those found in isolated words in Experiment 2 and the short sentences in Experiment 3 (as the medium sentences were identical in both experiments and elicited similar effects ${ }^{3}$ ). When we considered only medium utterances and short sequences (words in Experiment 2, short sentences in Experiment 3) and pooled the results across participants in both experiments, a significant by-participant effect of accent was found, $F_{1}(1,66)=12.21, p<.001$, and $F_{2}(1,18)=2.62, p=.12$, which interacted significantly with utterance length, $F_{1}(1,66)=$ $5.23, p=.025$, and $F_{2}(1,18)<1$. The triple interaction among utterance length, accent, and experiment was not significant, $F_{1}(1$, $66)<1$, and $F_{2}(1,18)<1$, showing that the difference in the effect of accent seen between medium sentences and shorter utterances was equivalent across experiments. Thus far, we have compared familiarly versus unfamiliarly accented utterances ranging from isolated words to long sentences in Experiments 2 and 3. Combining the results of both of these experiments indicates that the word identification disturbance due to an unfamiliar accent becomes more robust as more information is available from the speech signal.

Studies of foreign accent adaptation (e.g., Clarke \& Garrett, 2004; Gass \& Varonis, 1984) indicate that these processing costs should eventually drop and fall back to baseline processing after exposure to the accent has been sufficient to allow for complete adaptation. If this reversion holds for regional accent adaptation, then it would appear that even the longest sentences do not contain sufficient information for the participants to fully adapt to the unfamiliar regional accent. In this and all previous experiments,

\footnotetext{
${ }^{3}$ We verified that medium sentences elicited similar RTs in both experiments (mean RTs in Experiment 2: $581.8 \mathrm{~ms}$; in Experiment 3: $585.8 \mathrm{~ms}$ ), $F_{1}(1,66)<1$, and $F_{2}(1,18)<1$. Overall, there was a main effect of accent familiarity $F_{1}(1,66)=14.94, p<.001$, and $F_{2}(1,18)=1.96$, which did not interact significantly with the experiment, $F_{1}(1,66)<1$, and $F_{2}(1$, 18) $<1$.
} 
Table 3

Mean Reaction Times and Stimuli Duration (in Milliseconds) in Experiment 3

\begin{tabular}{cccc}
\hline Accent & $\begin{array}{c}\text { Word } \\
\text { duration }\end{array}$ & $\begin{array}{c}\text { Sentence } \\
\text { duration }\end{array}$ & $\begin{array}{c}\text { Mean } \\
\text { RT }\end{array}$ \\
\hline Familiar & & & \\
Speaker 1 & 487 & 1,819 & 560 \\
Speaker 2 & 494 & 1,440 & 558 \\
Unfamiliar & & & \\
Speaker 1 & 553 & 1,657 & 589 \\
Speaker 2 & 528 & 1,569 & 560 \\
\hline
\end{tabular}

Note. $\quad \mathrm{RT}=$ reaction time.

the accent of the sentences presented to the participants changed in an unpredictable manner, roughly every sentence. Therefore, it is likely that the change in accent from one sentence to the next disrupted the adaptation process before the associated costs could drop down to baseline levels. In Experiment 4, we evaluated this hypothesis by looking at longer term adaptation by blocking home and unfamiliar accents separately.

\section{Experiment 4}

In Experiment 4, we investigated the evolution of the word identification processing cost observed in the previous experiments by blocking home and unfamiliar accents separately. Under these conditions, we predicted no difference in RTs between the two blocks, as the processing cost associated with the unfamiliar accent should disappear during the presentation of the block due to complete adaptation. However, whether listeners fully recover their speech processing efficiency with a regional accent is still an open question, and RTs for the unfamiliar accent block could be still slower than for the home accent block.

In addition, to provide direct comparisons with foreign accent studies, we introduced a foreign accent condition in this experiment in which each participant heard a final block of Englishaccented long sentences. Lexical decision costs associated with that accent were expected to be higher than for the regional accent.

\section{Method}

Participants. Twenty participants (including 5 men) with an average age of 29 were tested in this experiment.

Stimuli. In addition to the stimuli previously used in Experiment 3, an additional block of 32 long sentences (17-19 syllables), one for each of the usual 16 targets, was recorded by two British women. Each of these women was an experienced French speaker but had acquired the language late in life (after puberty). Both had lived in the home accent region for 2 years prior to the experiment and were originally from south London.

Procedure. The procedure was similar to that used in previous experiments, diverging only in the blocking of accents, where sentences with home, unfamiliar, and foreign accents were grouped into separate blocks. For the home and unfamiliar accent groups, sentences were also blocked into length, with half of the participants presented with blocks in the order middle, short, long, and the others in the order middle, long, short. The order of home and unfamiliar accent blocks was also counterbalanced among the participants, with the foreign accent block always presented last. This organization of stimuli resulted in seven blocks, starting with 2 three-block (for the three lengths of sentences) groups of home and unfamiliar accented sentences, and a final block of sentences produced with a foreign accent. Between each of the accent groups, there was a pause of a few minutes.

\section{Results}

Out of the 2,800 required lexical decisions, 15 were missing and 54 were rejected as being too slow (RT above 2.5 times the standard deviation of each participant), leaving 2,731 lexical response times $(2.4 \%$ error rate). Errors due to slow RTs were predominantly found in foreign accented words (44\% of errors), rather than home (30\% errors) or unfamiliar (26\% errors) accents. For the 840 pseudowords, participants responded with 32 false alarms (3.8\% error rate).

ANOVAs of the RTs for home and unfamiliar accents were made with two between-participants variables, length presentation order (medium-short-long or medium-long-short) and accent presentation order (home-unfamiliar or unfamiliar-home). Three within-participant variables were considered: accent (home, unfamiliar, and foreign), speaker (two per accent), and sentence length (short, medium, and long). Summarizing significant $(p<.05)$ main effects and interactions, we found that, as in Experiment 3, sentence length, $F_{1}(2,32)=10.09, p<.001$, and $F_{2}(2,72)=$ $10.0, p<.001$, had a significant effect on participants' responses, with faster RTs for short $(581 \mathrm{~ms})$ and long sentences $(587 \mathrm{~ms})$ than medium sentences $(611 \mathrm{~ms})$. This effect is thought to be due to habituation, as the medium sentences were always presented first. Similarly, although accent presentation order had no main effect, $F_{1}(1,16)<1$, and $F_{2}(1,36)<1$, it did interact with accent, $F_{1}(1,16)=7.21, p=.016$, and $F_{2}(1,36)=24.97, p<.001$, showing that participants were faster during the presentation of the second accent than the first. On examination of the effect of speaker, we found that post hoc comparisons showed significant differences between speakers with home accent, $F_{1}(1,16)=$ $10.34, p<.001$, and $F_{2}(1,36)=3.69, p=.06$, and unfamiliarly accented sentences, $F_{1}(1,16)=17.71, p<.001$, and $F_{2}(1,36)=$ 10.37, $p<.001$, as can be seen in Table 4. Finally, it must be noted that responses, summarized in Figure 3, showed no significant main effects of regional accent, $F_{1}(1,16)<1$, and $F_{2}(1,36)<1$, nor, contrary to that found in Experiment 3, was there any interaction between accent and sentence length, $F_{1}(2,32)=1.80$, and $F_{2}(2,72)=1.5$. This interaction remained nonsignificant even

Table 4

Mean Target Word Duration, Sentence Duration, and Reaction Times for Experiment 4 (in Milliseconds)

\begin{tabular}{cccc}
\hline Accent & $\begin{array}{c}\text { Word } \\
\text { duration }\end{array}$ & $\begin{array}{c}\text { Mean } \\
\text { RT }\end{array}$ & $\begin{array}{c}\text { Sentence } \\
\text { duration }\end{array}$ \\
\hline Home & & & \\
$\quad$ Speaker 1 & 487 & 585 & 1,819 \\
$\quad$ Speaker 2 & 494 & 601 & 1,440 \\
Unfamiliar & & & \\
Speaker 1 & 553 & 611 & 1,657 \\
$\quad$ Speaker 2 & 528 & 579 & 1,569 \\
Foreign & & & \\
$\quad$ Speaker 1 & 675 & 732 & 4,317 \\
Speaker 2 & 821 & 701 & 4,142 \\
\hline
\end{tabular}

Note. $\quad \mathrm{RT}=$ reaction time. 


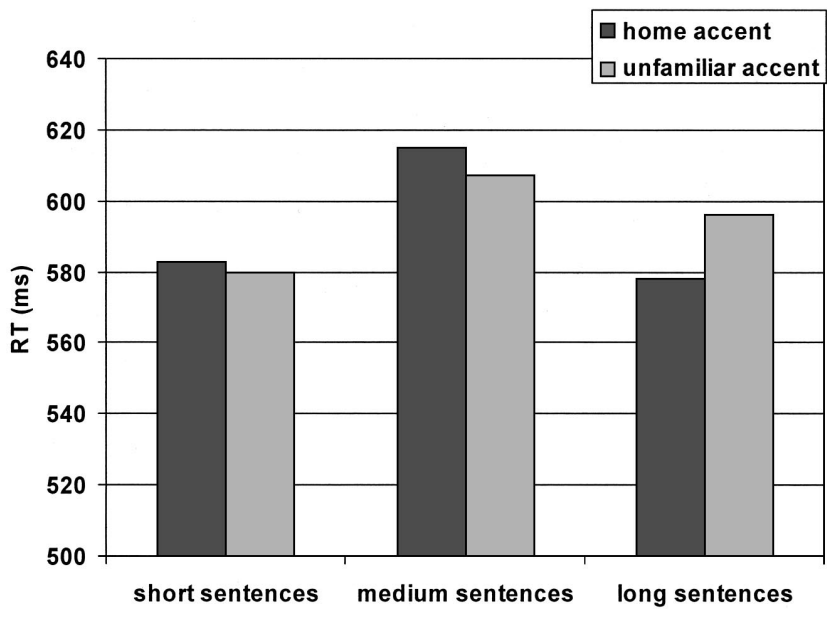

Figure 3. Mean reaction times (RTs) as a function of accent familiarity and sentence length in Experiment 4.

when the medium-length sentences were removed (because of the possible block order effect) and only short and long sentences were compared, $F_{1}(1,19)=3.61, p=.073$, and $F_{2}(1,9)=1.54$.

To assess the impact of experimental design (random presentation of accents as in Experiment 3 vs. blocked presentation as in Experiment 4) on the observation of the accent familiarity effect, we examined the interaction between accent (familiar vs. unfamiliar) and experiment (participants from Experiment 3 vs. Experiment 4), finding a significant interaction by participant, $F_{1}(1$, $53)=4.02, p=.045$, and $F_{2}(1,18)=2.32, p=.14$, showing that performance with an unfamiliar accent improved with a blocked design.

An additional ANOVA was made comparing the foreign accented sentence block with those of home and unfamiliar accents. This analysis revealed that participants were significantly slower with the foreign accent words (average of $716.9 \mathrm{~ms}$ ) than nativeFrench accents (average of $592.9 \mathrm{~ms}$ for home accent and average of $595.1 \mathrm{~ms}$ for unfamiliar accent), $F_{1}(2,32)=117.11, p<.001$, and $F_{2}(2,72)=192.54, p<.001$. This difference was still highly significant when only long sentence blocks were compared across accents.

As might be expected, the duration of foreign accented sentences (average of 4,230 ms) was much longer than those produced by French natives (average of 1,629 ms and 1,613 ms for home and unfamiliar accent long sentences, respectively). However, each of the accents showed a significant correlation between sentence duration and RTs (home accent: mean $B=0.017, t[19]=2.17$, $p=.04$; unfamiliar accent: $B=0.025, t[19]=4.01, p=.001$; foreign accent: $B=0.042, t[19]=2.83, p=.011)$. Analyses of target word duration and RTs showed that these factors were also significantly correlated for all accents (home accent: $B=0.27$, $t[19]=8.61, p=.000$; unfamiliar accent: $B=0.36, t[19]=7.41$, $p=.000$; foreign accent: $B=0.13, t[19]=5.09, p=.000)$. Therefore, if target or sentence duration differences were responsible for the foreign accent effect observed in this experiment, differences should have emerged between the home and the unfamiliar accent (see the duration of targets and sentences in Table 3).

Experiment 4 was not specifically designed to evaluate the time course of adaptation to regional accent; rather, it was designed for comparison with the findings of previous experiments under an accent blocking condition. However, post hoc analyses were conducted to examine the evolution of the word identification cost in each accent block across time. For each participant, we divided the medium sentence length blocks into three periods (10 first, 10 middle, and 12 last sentences). As can be seen in Figure 4, there was no robust evidence of initial disruption caused by unfamiliar accents, as RTs remained relatively constant across the three periods (main effect of period for each accent), $F_{1}(2,18)<1$. Similarly, the processing cost associated with the foreign accent remained constant across these three periods, $F_{1}(2,18)<1$.

Given that Clarke and Garrett (2004) found that the processing of a foreign accent returns to baseline performance after only $2-4$ sentences, we could reasonably assume that regional accent adaptation would occur over a similar time frame. However, in this experiment, the results of fine-grained analyses remain inconclusive because of the use of a go/no-go design. Because RT data

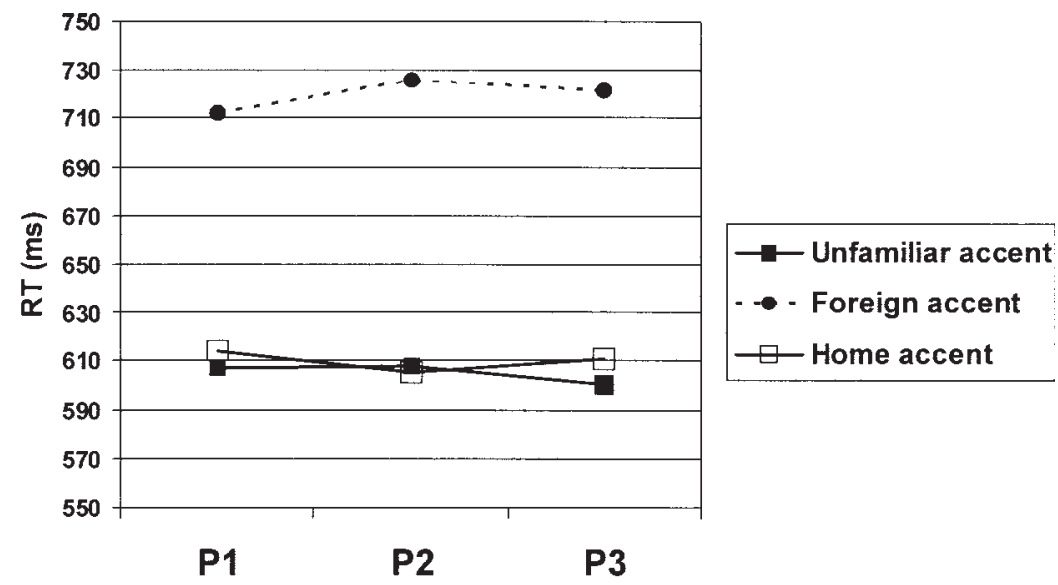

Figure 4. Evolution of reaction times (RTs) in Experiment 4 within the first presented block with home, unfamiliar, and foreign accent block. First period (P1): 10 initial sentences; second period (P2): 10 middle sentences; third period (P3): 12 final sentences. 
were available only from go responses, representing only two thirds of all responses, there were insufficient observations for a robust statistical examination of the evolution of RTs across individual sentences.

\section{Discussion}

In previous experiments, the maximum amount of accented speech offered for adaptation was usually no longer than 19 syllables in length. Over this time period, the processing costs due to adaptation were seen to rise steadily, resulting in a processing delay of around $30 \mathrm{~ms}$. In this experiment, we examined the effects of long-term adaptation by blocking accents separately, increasing the amount of uninterrupted accented speech by a factor of 32 . It appears that, with this design, there was no longer any significant difference between detection latencies for the unfamiliar and the home accent. When combined, these findings support our main hypothesis of regional accent normalization, that being a two stage process with initial word identification disruption, followed by eventual adaptation leading to a full recovery in comprehension speed.

However, this hypothesis also predicted that the very first presentation of the unfamiliar accent in Experiment 4 should elicit a word identification delay when compared with the familiar accent. Post hoc analyses failed to reveal such an effect, possibly because of the use of a go/no-go paradigm, which meant that a third of the possible responses at the moment of accent change were not recorded, thus reducing the power of sentence-by-sentence finegrained analysis. Further data will be needed to address the issue of the time course of full adaptation to a regional nonfamiliar accent (Floccia, Ellis, \& Goslin, 2006).

Data collected with the foreign accented speech showed that full accent adaptation is not always assured. As we have seen, the magnitude of the lexical decision delays due to this accent was much greater than that observed for regional accents (about $120 \mathrm{~ms}$ vs. $30 \mathrm{~ms}$, respectively) even after presenting participants with 32 sentences of uninterrupted accented speech. This result contrasts with those obtained by Clarke and Garrett (2004), who saw a return to baseline processing with foreign accents, and we will return to address the reasons for this disparity in the General Discussion.

Although the findings we have reported thus far appear relatively clear cut, supporting our main hypothesis on regional accent adaptation, two methodological issues remain, which could confound our conclusions. The first of these concerns the relatively small number of target words used in each of the experiments; in many cases, only 10 target words were used in each condition. Because participants were asked to respond to the same words on multiple occasions (as often as 12 times in Experiments 3 and 4), it is possible that this repetition could have an impact on their responses. The second issue relates to the generality of the unfamiliar accent effect we have found in our experiments. Thus far, we have assumed that the participants from Franche-Comté encountered word identification delays with the Toulouse accent because of their lack of familiarity to this accent. However, it is also possible that this effect was specific to the speaking style typified by this particular accent, rather than a product of unfamiliarity itself. Therefore, before advancing to the General Discussion, we present two experiments that address these issues.

\section{Experiment 5}

In this experiment, we examined the potential impact of target item repetition on the behavior of the participants in the previous experiments. In all of the experiments we have reported up to now, the participants have been asked to respond to the same word presented on multiple occasions. Because mass repetition is unusual in these types of experiments, it is possible that this design could have had a significant impact on the results reported thus far. For example, in Experiments 3 and 4, the repetition priming effect, found when comparing responses from the initial and later stimuli blocks, is larger than the accent effect. Although this effect could be due to task habituation, it is also possible that participants learned to predict the target words of the sentences, thus reducing RTs in subsequent repetitions. In this experiment, we addressed these concerns by attempting to replicate the regional accent familiarity effect seen in the previous experiments without using repeated target items.

\section{Method}

Participants. Twenty participants (including 2 men) with an average age of 19 years were tested in this experiment.

Stimuli. In this experiment, we reused all of the target words and pseudowords used in Experiments 1 and 3; items common to both experiments were only used once in this experiment. Each of these 28 words and 16 pseudowords formed the final word of two sentences: one produced in the home accent, the other in the unfamiliar accent. Four speakers were used to produce the sentences for each accent group, with unfamiliar accented sentences split between speakers from the Toulouse and Aix-enProvence $^{4}$ regions. Each of the sentences was arranged into two equalsized blocks, labeled $\mathrm{A}$ and $\mathrm{B}$, such that none of the target items were repeated within each block. In addition, the assignment of either the home or unfamiliar accented versions of each target item was randomly assigned between blocks such that each contained an equal mix of home and unfamiliar accented sentences. This meant that the participants heard each target item twice, once in both blocks, produced with a different accent each time. For example, the item bateau (boat) was presented in Block A in the home accent and in Block B in an unfamiliar accent, although piano was presented in Block A with an unfamiliar accent and in Block B with the home accent. Block presentation order was counterbalanced across participants.

Procedure. The procedure was the same as in Experiments 1-4.

\section{Results}

Out of the 1,120 required lexical decisions, 14 were missing, 24 were rejected as being too slow (RT greater than 2.5 times the standard deviation of RT for the participant), and 11 were rejected for anticipation (RT below 2.5 times the standard deviation or inferior to $300 \mathrm{~ms}$ ), leaving 1,071 lexical response times $(4.4 \%$ error rate). For the 640 pseudowords, participants responded with 37 false alarms $(5.8 \%)$.

Mean RTs were examined using ANOVA. These analyses were conducted with three within-participant/item variables: accent (home vs. unfamiliar), speaker (four per accent), and block (A vs.

\footnotetext{
${ }^{4}$ Post hoc analyses showed that the origins of the speakers (Toulouse or Aix) in the unfamiliar accent condition of Experiment 5 did not have any significant effect, as if listeners classified them as belonging to a broad southern category.
} 
B). A between-participants condition was also used to measure the effect of block order (either A-B or B-A). These analyses revealed that RTs were significantly faster with the home accent than the unfamiliar accent (709.4 vs. $740.3 \mathrm{~ms}), F_{1}(1,18)=9.78, p=.006$, and $F_{2}(1,26)=3.64, p=.067$, and that there was no significant interaction between this factor and the stimuli block (A or B), $F_{1}(1,18)=1.96$, and $F_{2}(1,26)<1$. The main effect of block was also found to be significant, $F_{1}(1,18)=8.05, p=.01$, and $F_{2}(1$, $26)=1.14$, with RTs faster for Block A (700.2 ms) than Block B (734.7 ms). As to the possible effects of target item repetition, we found that the between-participants effect of block order did not have any significant effect, $F_{1}(1,18)=1.15$, on RTs nor did it significantly interact with accent, $F_{1}(1,18)<1$. Additionally, no significant interaction was found among the factors of accent, block, and block order, $F_{1}(1,18)<1$, showing that there was no significant difference in the magnitude of the accent effect between the first $(26.3 \mathrm{~ms})$ and second $(30.5 \mathrm{~ms})$ presentation of the target items. Finally, significant effects of individual speakers were found for both accents (home accent: $F_{1}[3,54]=10.80, p<$ .001 , and $F_{2}[3,52]=1.06$; unfamiliar accent: $F_{2}[3,54]=52.61$, $p<.001$, and $F_{2}[3,52]=6.37, p<.001$ ), as shown in Table 5 .

Further regression analyses of individual coefficients revealed that sentence length had no significant effect over RTs (mean $B=$ $-0.003), t(39)<1$, although target word length effect was significant (mean $B=0.14), t(39)=3.53, p=.001$. However, as can be seen in Table 5 , the duration of targets produced by the speakers with different accents was highly similar, $t(27)=1.23$; therefore, it is unlikely that the effect of accent familiarity was due to differences in stimuli duration.

\section{Discussion}

The main aim of this experiment was to verify that the findings of the previous four experiments were not being colored by effects arising from the repetition of target items. This experiment explicitly tested the possible effects of repetition on unfamiliar accent processing by comparing the costs associated with this adaptation between the first and second presentation of target items. Our findings reveal that the processing delay resulting from unfamiliar

Table 5

Mean Sentence Duration (up to the Beginning of the Target Word), Mean Word Duration, and Mean Associated Reaction Times, as a Function of Accent and Speaker (in Milliseconds) in Experiment 5

\begin{tabular}{cccc}
\hline Accent & $\begin{array}{c}\text { Sentence } \\
\text { duration }\end{array}$ & $\begin{array}{c}\text { Word } \\
\text { duration }\end{array}$ & RT \\
\hline Home & & & \\
Speaker 1 & 2,518 & 488 & 660 \\
Speaker 2 & 1,981 & 499 & 701 \\
Speaker 3 & 2,272 & 536 & 722 \\
Speaker 4 & 1,605 & 473 & 726 \\
Unfamiliar & & & 730 \\
Speaker 1 & 2,475 & 546 & 642 \\
Speaker 2 & 2,103 & 525 & 769 \\
Speaker 3 & 2,279 & 530 & \\
Speaker 4 & 2,221 & 514 & \\
\hline
\end{tabular}

Note. $\quad \mathrm{RT}=$ reaction time. accents was not significantly affected by repetition. This would rule out the possibility that the previously obtained accent adaptation effects could be due to learning effects from target item repetition.

\section{Experiment 6}

Throughout this study, we have assumed that the word identification delay encountered by our participants when faced with the Toulouse accent was due to its unfamiliarity. However, an alternative interpretation of these findings is that they are due to features specific to the Toulouse accent, rather than to generic accent adaptation. In this experiment, we evaluated these alternative explanations by reversing the conditions used in our previous experiments, that is, by testing participants native to Toulouse with their own accent and alternative unfamiliar accents. If the effects seen in the previous experiments were due to specific effects of the Toulouse accent, then we would predict RT delays similar to those we have seen in the previous experiments, that is, longest RTs for the Toulouse accent. On the other hand, generic effects resulting from adaptation to unfamiliar accents would result in a reverse pattern to that observed in the previous experiments, that is, shortest RTs for the now-native Toulouse accent.

One of the unfamiliar accents chosen for the Toulouse participants was that of Franche-Comte, the native region of the participants from the previous experiments. However, a potential problem with the Franche-Comté accent is that it may not be distinct enough from the Parisian accent, used as a familiar accent in Experiment 1. In that experiment, there was no significant difference found between the processing of the participants' native Franche-Comté accent and that of the Parisian accent. Therefore, in addition to the Franche-Comté accent, we have introduced another unfamiliar accent, that of the francophone Swiss, which is perceptually more distant from the Parisian accent than that of Franche-Comté (Carton et al., 1983). This accent belongs to the Franco-Provençal dialect set, whereas the Franche-Comté accent is related to the dialects of Oil, in common with the standard massmedia accent (Battye et al., 2000; Rash, 2002).

\section{Method}

Participants. Nineteen participants (including 6 men) with an average age of 23 years 5 months were tested in this experiment. The data of 1 additional participant were removed because of a high rate of false alarms (more than 20\%). All of these participants were tested in the Toulouse region and were selected using the same criteria as those from the previous experiments, except that their home region and accent should now be Toulouse.

Stimuli. In this experiment, we reused the long sentences previously tested in Experiments 3 and 4 for the home (then unfamiliar) and unfamiliar Franche-Comté (then home) accents. Additional sentences were recorded for the unfamiliar Swiss French accent by two native female monolingual French speakers from Fribourg, a Swiss canton where the majority of the population are French speaking. As in Experiments 3 and 4 , each speaker produced one sentence for each of the 10-word and 6-pseudoword targets.

Procedure. The procedure was the same as in Experiment 3 but with only one block of 96 long sentences randomly presented to the participants. 


\section{Results}

Out of the 1,140 required lexical decisions, 18 were missing and 29 were rejected as being too slow (RT above 2.5 times the standard deviation of each participant), leaving 1,093 lexical response times ( $4.1 \%$ error rate). For the 684 pseudowords, participants responded with 34 false alarms (5.0\%).

ANOVAs of the RTs were conducted with two withinparticipant variables: accent (home, unfamiliar Franche-Comté, and unfamiliar Swiss French) and speaker (two per accent). These analyses revealed an effect of speaker with the home accent but not with either of the unfamiliar accents (home accent: $F_{1}[1,18]=$ $6.88, p=.02$, and $F_{2}[1,9]=2.60$; unfamiliar Franche-Comté: $F_{1}[1,18]=2.24$, and $F_{2}[1,9]<1$; unfamiliar Swiss French: $F_{1}[1$, $18]=1.81$, and $F_{2}[1,9]<1$ ). The factor of regional accent was found to have a strong significant effect in this experiment, $F_{1}(2$, $17)=24.94, p<.001$, and $F_{2}(2,8)=12.17, p=.004$, with faster lexical decisions in the home accent than the unfamiliar accents (664.6 ms vs. $690.7 \mathrm{~ms}$, respectively). Further analyses revealed that this effect was mainly due to the Swiss French accent, with average RTs of $717.9 \mathrm{~ms}$ compared with the Franche-Comté accent with $663.5 \mathrm{~ms}$ (home accent vs. unfamiliar Franche-Comté accent: $F_{1}[1,18]<1$, and $F_{2}[1,9]<1$; home accent vs. unfamiliar Swiss French accent: $F_{1}[1,19]=48.19, p<.001$, and $F_{2}[1$, 9] $=26.28, p=.001)$.

The behavior of Franche-Comté listeners in Experiment 3 was directly compared with those of Toulouse in this experiment by examining possible interactions between participant group and accent (Franche-Comté vs. Toulouse). The outcome of this analysis was found to be significant by participant, $F_{1}(1,52)=6.68$, $p=.013$, and $F_{2}(1,28)=3.52, p=.071$, showing that the two groups of participants displayed different behaviors with the same accents.

Regression of RTs and sentence duration were found to be significant for the home accent only (mean $B=0.055), t(18)=$ 2.87, $p=.01$, although similar analyses for target word length were significant across all accents (home accent: mean $B=0.17$, $t[18]=2.34, p=.03$; unfamiliar Franche-Comté accent: mean $B=0.48, t[18]=7.37, p<.001$; unfamiliar Franche-Comté accent: mean $B=0.40, t[18]=6.65, p<.001$ ), with longer words eliciting slower RTs. Table 6 shows that the words produced by

Table 6

Mean Sentence Duration, Word Duration, and Reaction Times as a Function of Accent and Speakers (in Milliseconds) in Experiment 6

\begin{tabular}{lccc}
\hline \multicolumn{1}{c}{ Accent } & $\begin{array}{c}\text { Sentence } \\
\text { duration }\end{array}$ & $\begin{array}{c}\text { Word } \\
\text { duration }\end{array}$ & $\begin{array}{c}\text { Mean } \\
\text { RT }\end{array}$ \\
\hline Home & & & \\
$\quad$ Speaker 1 & 2,382 & 531 & 681 \\
$\quad$ Speaker 2 & 2,099 & 518 & 648 \\
Unfamiliar (Franche-Comté) & & & \\
$\quad$ Speaker 1 & 2,470 & 492 & 654 \\
$\quad$ Speaker 2 & 1,996 & 492 & 673 \\
Unfamiliar (Swiss French) & & & \\
$\quad$ Speaker 1 & 3,264 & 643 & 708 \\
$\quad$ Speaker 2 & 2,931 & 568 & 727 \\
\hline
\end{tabular}

Note. $\quad \mathrm{RT}=$ reaction time.
Swiss speakers were significantly longer than those produced by the home accent speakers, $t(19)=4.18, p<.001$, which could explain why participants were slower to identify words in this accent. However, comparisons between the Swiss speakers revealed that the speaker who produced the longest words (643 vs. $568 \mathrm{~ms}$ ), $t(9)=3.67, p=.005$, elicited the fastest RTs (708 vs. $727 \mathrm{~ms}$ ). Therefore, it seems unlikely that the slow RTs elicited by the Swiss unfamiliar accent were purely due to durational differences.

\section{Discussion}

Experiment 6 was designed to determine whether the accent effects obtained in the preceding experiments were due to participants' familiarity with the accents or to a specific difficulty in processing the speech local to Toulouse. In this experiment, participants from Toulouse were presented with their own, home, accent plus two unfamiliar accents from the Franche-Comté and French-speaking Swiss regions. Analyses of RT differences elicited by these accents revealed that participants from the Toulouse region had significantly different responses to the two unfamiliar accents. In previous experiments, natives of the Franche-Comté region encountered comprehension delays when processing the Toulouse accent. However, in this experiment, participants from Toulouse had no greater difficulty processing sentences produced by the Franche-Comté accent than their own native accent. In contrast, when sentences were produced in the Swiss French accent, the participants were significantly slower to react to target items than they were in their native Toulouse accent. This result clearly shows that the word identification deficits seen in this and prior experiments are due to the accent unfamiliarity rather than to specific aspects of speech style used in Toulouse.

As stated in the introduction of this experiment, the perceptual distance between the Franche-Comté accent and that of the Parisian, mass-media, accent is relatively narrow. This would explain why our participants were relatively familiar with its idiosyncrasies and thus did not suffer from any processing delays due to adaptation. However, the Swiss French accent has far less in common with the Parisian accent, and although most French people might be able to recognize a stereotypical Swiss French accent, exposure to this accent would have been minimal. For example, the Swiss French accent is typified by systematic paroxytonie (accentuation of nonfinal syllables) as opposed to the oxytonie of Parisian French (accentuation of the last syllable) Although this property is also evident in the Franche-Comté accent, it is not systematic and thus is far less frequent than in the Swiss French accent (Carton et al., 1983).

\section{General Discussion}

The main findings of this study, summarized in Table 7, indicate that regional accent normalization is typified by an initial temporary perturbation in speech processing, which becomes evident after only a certain amount of accented signal has been processed. In our initial experiment, we found that when an unfamiliar southern French accent was presented to participants from the FrancheComté region, word identification was delayed by approximately $30 \mathrm{~ms}$. Experiments 2 and 3 explored the amount of signal necessary to obtain this processing cost, with Experiment 3 revealing 
Table 7

Summary of the Six Experimental Designs and Associated Results

\begin{tabular}{|c|c|c|c|c|}
\hline Experiment & Accents & Design & Utterance length & Accent effect \\
\hline 1 & Home, familiar, unfamiliar & Exploratory & Not controlled & $33 \mathrm{~ms}$ \\
\hline 2 & Home, unfamiliar & Isolated words & $\begin{array}{l}\text { Isolated words and } \\
\text { middle-length sentences }\end{array}$ & Overall: $11 \mathrm{~ms}$ \\
\hline 3 & Home, unfamiliar & $\begin{array}{l}\text { Manipulation of carrier } \\
\text { sentence length }\end{array}$ & Short, middle, long & $\begin{array}{l}\text { Short: none } \\
\text { Middle: } 13 \mathrm{~ms} \\
\text { Long: } 27 \mathrm{~ms}\end{array}$ \\
\hline 4 & Home, unfamiliar, foreign & Blocking of accents & Short, middle, long & $\begin{array}{l}\text { Home versus unfamiliar: None } \\
\text { Foreign versus the others: } 123 \mathrm{~ms}\end{array}$ \\
\hline 5 & Home, unfamiliar & $\begin{array}{l}\text { No repetition in the first } \\
\text { block }\end{array}$ & Long & $31 \mathrm{~ms}$ \\
\hline 6 & $\begin{array}{l}\text { Home, FC unfamiliar, SF } \\
\text { unfamiliar }\end{array}$ & Participants from Toulouse & Long & $\begin{array}{l}\text { Home vs. FC unfamiliar: None } \\
\text { Home vs. SF unfamiliar: } 53 \mathrm{~ms}\end{array}$ \\
\hline
\end{tabular}

Note. $\quad \mathrm{FC}=$ Franche-Comté; $\mathrm{SF}=$ Swiss French.

rising adaptation as the amount of accented signal presented to the participant increased. In the early stages of this process, with short sentences of between seven and nine syllables, the disruption caused by this process was below significance. However, when between 12 and 14 syllables of accented speech had been presented to the participants, the processing delay became significant and subsequently increased with longer sentences. In Experiment 4 , we established that the disruption to word identification was not observed under blocked presentation of accents, suggesting that after sufficient information on the accent has been gathered, comprehension returns to baseline levels, indicating full adaptation. However, this last conclusion must be taken cautiously, because post hoc analyses failed to demonstrate any initial disruption of word identification immediately following accent change, although this could have been due to the low power of the go/no-go design.

The final two experiments were conducted to investigate two methodological concerns that arose from the design of the previous experiments. In all of the previous experiments, the same target words and pseudowords were presented to the participants on multiple occasions, raising the possibility that the results of these experiments could have been colored by repetition effects. In Experiment 5, we addressed this concern by comparing unfamiliar accent adaptation on the first and the second presentation of target items. This experiment revealed that repetition had no significant effect on the accent-related comprehension delays that were noted in previous experiments. The second issue relates to the generality of the unfamiliar accent effect we have found in our experiments. In most of the experiments, the unfamiliar accented conditions were represented by a single accent, that of the Toulouse region. Therefore, it was possible that the effects previously ascribed to general effects of accent unfamiliarity could have been specific to the speaking style of this region, rather than to a product of unfamiliarity itself. This potential confound was investigated in Experiment 6, where we reversed the conditions of the previous experiments presenting participants from Toulouse with accents from Franche-Comte and the French-speaking region of Switzerland. The results of this experiment revealed that these participants had no greater difficulty in processing the Franche-Comté accent than they did their own, although they did show the previously noted unfamiliarity effect with the Swiss accent. These findings indicate that the participants were able to assimilate the FrancheComté with the familiar Parisian accent, an accent that did not incur an adaptation cost in listeners from Franche-Comté either (see Experiment 1). However, adaptation was required for the Swiss accent, as it was not familiar to the participants nor was it perceptually similar to any other familiar accent. These results show that the processing delay caused by regional accent adaptation is not specific to any particular speaking style; rather, it is the result of listeners' unfamiliarity with an accent. Later in this discussion, we examine the implications of the initial disruption of word identification following unfamiliar accent presentation to theoretical accounts of accent normalization.

Another potential concern for the conclusions of this study lies in the robustness of the accent effects presented in this study and whether they may simply be ascribed to individual differences between speakers, rather than to accent. Although recurrent within-accent speaker effects were found between Experiments 2 and 6 , these effects were highly inconsistent. In some experiments, interspeaker differences were found between the speakers representing the home accent (Experiments 2 and 5), although there were differences between speakers representing unfamiliar accents (Experiments 3 and 5). It is this inconsistency, especially when compared with the consistent and significant effects of accent seen throughout our experiments, which lead us to ascribe interspeaker differences as sampling effects. To test this supposition, we examined the consistency of accent and speaker effects across experiments, comparing the RTs of Franche-Comté participants when presented with the same two speakers of the Toulouse and home accents. Under these conditions, participant responses were pooled from Experiment 2 (middle-length sentences), Experiment 3 (middle and long sentences), and Experiment 5 to form a between-participants factor of a repeated measures ANOVA, with within-participant factors of accent (Franche-Comté vs. Toulouse) and speaker (two per accent). This analysis revealed a main effect of accent, $F_{1}(1,85)=12.48, p=.001$, which, most important, did not interact with the between-participants factor of experiment, $F(2,85)=1.06$. On the other hand, although speaker effects were found across both accents (home accent: $F_{1}[1,85]=12.02, p=$ .001 ; unfamiliar accent: $\left.F_{1}[1,85]=81.06, p<.000\right)$, this effect was found to interact with the factor of experiment (home accent: $F_{1}[2,85]=4.86, p=.01$; unfamiliar accent: $F_{1}[2,85]=30.59$, 
$p<.000)$. This analysis indicates that the accent effect observed with Toulouse and Franche-Comté speakers is highly robust and can be generalized across speakers of the same region. This effect can be compared with the variation arising between the speakers of the two accents, which was found to change between experiments and is therefore most likely to be due to a simple sampling effect.

\section{Disrupting Word Identification}

According to Johnson's (1997) model of lexical access, speech variability is an inherent part of the stored exemplars used to represent words. However, by definition, an unfamiliar accent will contain variability that falls outside of the listener's experience and will not be encapsulated within the stored exemplars. In this case, the partial mismatch between stored exemplars and the incoming accented speech would reduce the efficiency of word recognition and thus lead to increased RTs. However, as more accented speech is presented to the listener, this would cause a progressive shift in the weights used to represent the relevant dimensions of the signal, finally leading to full adaptation. According to the model of lexical feedback proposed by Norris et al. (2003), mismatches between incoming phonological forms and those sorted in the lexicon will trigger training signals back to the prelexical level to signal adaptation. As in the previous model, this mismatch will initially result in poor lexical activation until the creation of a filter capable of matching the incoming phonemic forms onto those used in the lexicon.

\section{Initial Disruption and Utterance Length}

One finding of particular note arising from this study was the amount of accented speech required before the observation of a robust comprehension deficit. It is unlikely that the first 10-15 syllables of an utterance were bereft of cues indicating the use of an unfamiliar accent, as the participants reported that they were able to detect these accents even in isolated words. Additional analyses conducted by trained phoneticians also revealed that systematic pitch and rhythmic differences were evident between accented sentences, showing that accent-related prosodic patterns were present in all lengths of utterances. Unfortunately, it is difficult, if not impossible, to quantify all of the accent-related variations found in speech, a reason why most research in this domain is focused on phonemic contrasts, rather than on the perception of continuous speech. However, it is not unreasonable to suppose that the longer the utterance, the more accent-related information is revealed to the listener. How can the two described models of accent processing account for this finding?

One possible explanation for this progressive deterioration, which holds for the two types of models, is that as lexical activation becomes weaker, checking procedures are used to examine possible reasons for this failure. As these procedures are likely to consume both time and resources, they could progressively slow the lexical identification device. Another potential explanation stems from a weakening of feedback from higher levels of language processing caused by a weakening in the spread of activation from lexical levels. In this case, the progressive deterioration in lexical access is explained by a circular spread of weaker activation from both bottom-up and top-down processing.
Perhaps a more viable alternative explanation for progressive word identification disruption stems from the finding that participants are aware of unfamiliar accents well before any associated processing costs are evident. This leads us to suggest that this processing cost is not directly associated with a reduction in lexical activation, but rather it is an indication of a general slowing in the speech perception system. This slowing is caused by the selection and retrieval of an appropriate accent model from long-term memory containing phonological, phonotactic, and prosodic details about the previously encountered accents. This selection process would require the listener to extract information about the accent and to make a comparison with those already stored in memory. Therefore, as more information is gathered about an accent, the complexity of cross-checking stored entries increases, resulting in a progressive slowing of the speech processing system. Finally, when sufficient information has been gathered to make a match, the required representation can be retrieved from memory and used to adapt to the incoming signal.

\section{Comparing Regional and Foreign Accents}

According to Clarke and Garrett (2004), accents can be ranked on a perceptual scale as a function of their distance to the home dialect. They also proposed that the same processes are used for both accented and nonaccented speech and that differences observed between accents are due to exaggeration of these processes. This suggests that both the time course and amplitude of accentrelated effects should be similar in foreign and regional accents, with a simple modulation reflecting their relative distance to the native accent. Our observations appear to support this hypothesis, at least over the initial stages of normalization, as the word identification delays caused by regional accents were found to be smaller for regional (30 ms) accents than foreign accents (100-150 $\mathrm{ms})$. However, our findings diverge from those predicted by Clarke and Garrett when we consider the differences we observed in the time course of normalization for foreign accents. Participants in Clarke and Garrett's study were sometimes found to return to baseline processing levels after the presentation of only 2-4 sentences of foreign accented speech, indicating that they had achieved full adaptation. However, in our study, we found that the processing deficit observed with a foreign accent did not reduce over time, even after the presentation of a contiguous block of 32 sentences. A possible explanation for the disparity in foreign accent adaptation could lie in our use of multiple speakers for each of the accents, whereas the foreign accented speech used by Clarke and Garrett was produced by only a single speaker. For complete adaptation to take place in our experiment, it would have been necessary for listeners to transfer adaptation across the two speakers.

A number of studies have examined the transfer of foreign accent adaptation across speakers, with mixed results. Gass and Varonis (1984) reported that recognition accuracy in foreign accented sentences improved after exposure to different speakers of the same accent when compared with exposure to different foreign accents, indicating a possible transfer of adaptation across speakers. In a study by Weil (2001), English speakers were trained with words, sentences, and prose uttered by a native of Marathi. It was found that the resultant adaptation only transferred across to another Marathi speaker when the participants were presented with a 
certain sentence length utterances. According to Jongman, Wade, and Sereno (2003), this failure may have been due to the training task's emphasis on differences rather than on similarities across speakers. Still, Jongman et al. hypothesized that for robust learning effects to occur, participants would require exposure to multiple speakers of a given foreign accent. To test this hypothesis, they exposed English listeners to Spanish-accented English words uttered by many different speakers. However, contrary to their predictions, no improvement was reported when the participants were presented with new Spanish-accented words uttered by unfamiliar speakers (see also Clarke, 2000).

In light of this evidence, it is clear that the transfer of foreign accent adaptation is far from being assured and as such could account for the failure of our participants to fully adapt to foreign accent. Because two speakers were used to produce the sentences in Experiment 4, it is possible that the subsequent changes in speaker within the block of 32 sentences could have disrupted adaptation. These findings are in stark contrast to those found with regional accents, where long-term habituation to unfamiliar accents was found under similar conditions. As each block was represented by a random mix of sentences produced by two speakers, it is clear that listeners must have been able to transfer regional accent adaptation between speakers for this habituation to take place.

Although highlighting fundamental differences between adaptation mechanisms, the disparity between the transfer of regional and foreign accent adaptation also has important implications for models of lexical access. The architecture of the Johnson (1997) exemplar-based model predicts that accent adaptation should transfer across speakers with either foreign or regional accents. When a speaker is encountered with an unfamiliar accent, the weights used to represent multiple dimensions should be modified accordingly. However, even when a new speaker is presented, the weight changes previously instigated should still be relevant to the processing of the accent; therefore, there should not be any significant disruption to adaptation. In the model of Norris et al. (2003), the use of abstract prelexical representations should ensure than any within-speaker differences within the same accent should be normalized at the prelexical level of processing. That is, the acoustic speaker-related information should already have been removed before the phonological representation is used to access the lexicon. Therefore, any adaptation to existing prelexical representations resulting from accented speech should be relevant to all speakers within that accent. Although this may be the case for regional accents, which we can expect to be relatively homogeneous, foreign accents are more likely to be highly speaker specific. For nonnative speakers, differences in the levels of fluency or linguistic experience can have a significant impact on their production of the language. The phonetic realizations of particular phonemes produced by these speakers can range from their own native representations toward those used in the particular region in which they learned the language. Therefore, it is possible that interspeaker differences are greater than those found within the same foreign accent, meaning that the resulting adaptation is not general to the accent but specific to the speaker.

In conclusion, the main findings of this study point toward a normalization mechanism that causes an increasing delay in word identification latency, although information is gathered on the unfamiliar accent. Further research will be required to gain a fuller understanding on the mechanisms by which provision of sufficient accented speech should eventually lead to full adaptation and a return to baseline speech processing performance, allowing researchers to explain how humans' language comprehension system adapts to accent variation.

\section{References}

Altmann, G. T. M., \& Young, D. H. (1993). Factors affecting adaptation to time-compressed speech. Eurospeech '93, 333-336.

Battye, A., Hintze, M.-A., \& Rowlett, P. (2000). The French language today: A linguistic introduction (2nd ed.). New York: Routledge.

Best, C. T., McRoberts, G. W., \& Goodell. E. (2001). American listeners' perception of nonnative consonant contrasts varying in perceptual assimilation to English phonology. Journal of the Acoustical Society of America, 109, 775-794.

Bradlow, A. R., \& Bent, T. (2003). Listener adaptation to foreign accented English. In M. J. Sole, D. Recasens, \& J. Romero (Eds.), Proceedings of the XVth International Congress of Phonetic Sciences, Barcelona, Spain (pp. 2881-2884). Barcelona, Spain: Universitat Autonoma de Barcelona.

Bradlow, A. R., Nygaard, L. C., \& Pisoni, D. B. (1999). Effects of talker, rate, and amplitude variation on recognition memory for spoken words. Perception \& Psychophysics, 61, 206-219.

Carton, F., Rossi, M., Autesserre, D., \& Léon, P. (1983). Les Accents du Français [Accents of French]. Paris: Hachette, De Bouche à Oreille.

Clarke, C. M. (2000). Perceptual adjustment to foreign-accented English. Journal of the Acoustical Society of America, 107, 2856.

Clarke, C. M., \& Garrett, M. F. (2004). Rapid adaptation to foreignaccented English. Journal of the Acoustical Society of America, 116, 3647-3658.

Dupoux, E., \& Green, K. (1997). Perceptual adjustment to highly compressed speech: Effects of talker and rate changes. Journal of Experimental Psychology: Human Perception and Performance, 23, 914-927.

Dupoux, E., Pallier, C., Sebastián-Gallés, N., \& Mehler, J. (1997). A destressing "deafness" in French? Journal of Memory and Language, 36, 406-421.

Durand, J., Laks, B., \& Lyche, C. (Eds.). (2002). Phonologie du Français Contemporain, Bulletin $n^{\circ} 1$, Protocole, conventions et directions $d^{\prime}$ analyse [Phonology of Contemporary French. Bulletin ${ }^{\circ} 1$, Protocol, Conventions and Direction for Analysis]. Toulouse, France: Centre National de la Recherche Scientifique and University of Toulouse-Le Mirail.

Evans, B. G., \& Iverson, P. (2004). Vowel normalization for accent: An investigation of best exemplar locations in northern and southern British English sentences. Journal of the Acoustical Society of America, 115, $352-361$.

Flege, J. (1995). Second language speech learning: Theory, findings and problems. In W. Strange (Ed.), Speech perception and linguistic experience: Theoretical and methodological issues (pp. 233-277). Baltimore: York Press.

Floccia, C., Ellis, L., \& Goslin, J. (2006). Time-course of adaptation to regional and foreign accents. Manuscript in preparation.

Floccia, C., Kolinsky, R., Dodane, C., \& Morais, J. (2003). Discriminating spoken words in French: The role of the syllable and the CV phonological skeleton. Language and Cognitive Processes, 18, 241-267.

Gass, S., \& Varonis, E. (1984). The effect of familiarity on the comprehensibility of nonnative speech. Language Learning, 34, 66-85.

Goldinger, S. D. (1996). Words and voices: Episodic traces in spoken word identification and recognition memory. Journal of Experimental Psychology: Learning, Memory, and Cognition, 22, 1166-1183.

Hintze, M.-A., Pooley, T., \& Judge, A. (2001). French accents: Phonological and sociolinguistic perspectives. Location: National Centre for Languages-Association for French Language Studies.

Johnson, K. (1997). Speech perception without speaker normalization: An 
exemplar model. In K. Johnson \& J. Mullenix (Eds.), Talker variability in speech processing (pp. 145-166). San Diego, CA: Academic Press.

Jongman, A., Wade, T., \& Sereno, J. (2003). On improving the perception of foreign-accented speech. In M. J. Sole, D. Recasens, \& J. Romero (Eds.), Proceedings of the 15th International Congress of Phonetic Sciences (pp. 1561-1564). Barcelona, Spain: Universitat Autonoma de Barcelona.

Jusczyk, P. W., \& Luce, P. A. (2002). Speech perception and spoken word recognition: Past and present. Ear and Hearing, 23, 2-40.

Klatt, D. (1981). Lexical representations for speech production and perception. In T. Meyers, J. Laver, \& J. Anderson (Eds.), The cognitive representation of speech (pp. 11-31). Amsterdam: North-Holland.

Knecht, P., \& Rubattel, C. (1984). A propos de la dimension sociolinguistique du français en suisse romande [About the sociolinguistic dimension of French in French-speaking Switzerland]. Le Français Moderne, $52,138-150$.

Kolinsky, R. (1998). Spoken word recognition: A stage processing approach to language differences. European Journal of Cognitive Psychology, 10, 1-40.

Konopczynski, G. (1979). Description phonétique et phonologique du français régional de Besançon [Phonetic and phonological analysis of the variety of French spoken in Besançon]. Cahiers du CRELEF, 4, $2-19$.

Labov, W. (1989). The limitations of context: Evidence from misunderstandings in Chicago. Chicago Linguistics Society, 25, 171-200.

Lahiri, A., \& Marslen-Wilson, W. (1991). The mental representation of lexical form: A phonological approach to the recognition lexicon. Cognition, 38, 245-294.

Lane, H. (1963). Foreign accent and speech distortion. Journal of the Acoustical Society of America, 35, 451-453.

Lorch, R. F., \& Myers, J. L. (1990). Regression analyses of repeated measures data in cognitive research. Journal of Experimental Psychology: Learning, Memory, and Cognition, 16, 149-157.

Martin, C. S., Mullennix, J. W., Pisoni, D. B., \& Summers, W. V. (1989). Effects of talker variability on recall of spoken word lists. Journal of Experimental Psychology: Learning, Memory, and Cognition, 15, 676684

Matthey, M. (2003). Le français langue de contact en Suisse Romande [French as a contact language in French-speaking Switzerland]. Glottopol, 2, Retrieved July 31, 2006, from http://www.univ-rouen.fr/ dyalang/glottopol/telecharger/numero_2/09matthey.pdf

McClelland, J. L., \& Elman, J. L. (1986). The TRACE model of speech perception. Cognitive Psychology, 18, 1-86.

McLennan, C. T., \& Luce, P. A. (2005). Examining the time course of indexical specificity effects in spoken word recognition. Journal of Experimental Psychology: Learning, Memory, and Cognition, 31, 306321.

Mehler, J., Dommergues, J.-Y., Frauenfelder, U. H., \& Segui, J. (1981). The syllable's role in speech segmentation. Journal of Verbal Learning and Verbal Behavior, 20, 298-305.

Mullennix, J. W., Pisoni, D. B., \& Martin, C. S. (1988). Some effects of talker variability on spoken word recognition. Journal of the Acoustical Society of America, 85, 365-378.

Munro, M. J., \& Derwing, T. G. (1995). Processing time, accent and comprehensibility in the perception of native and foreign-accented speech. Language and Speech, 38, 289-306.

Munro, M. J., \& Derwing, T. G. (1999). Foreign accent, comprehensibility and intelligibility in the speech of second language learners. Language Learning, 49, 285-310.
New, B., Pallier, C., Ferrand, L., \& Matos, R. (2001). Une base de données lexicales du français contemporain sur internet: LEXIQUE [An online lexical database for contemporary French: LEXIQUE]. L'Année Psychologique, 101, 447-462.

Norris, D. (1994). Shortlist: A connectionist model of continuous speech recognition. Cognition, 52, 189-234.

Norris, D., McQueen, J. M., \& Cutler, A. (2003). Perceptual learning in speech. Cognitive Psychology, 47, 204-238.

Pallier, C., Colomé, A., \& Sebastián-Gallés, N. (2001). The influence of native-language phonology on lexical access. Psychological Science, 12, 445-449.

Pallier, C., Dupoux, E., \& Jeannin, X. (1997). EXPE: An expandable programming language for on-line psychological experiments. Behavior Research Methods, Instruments, and Computers, 29, 322-327.

Pallier, C., Sebastián-Gallés, N., Dupoux, E., Christophe, A., \& Mehler, J. (1998). Perceptual adjustment to time-compressed speech: A crosslinguistic study. Memory and Cognition, 26, 844-851.

Pisoni, D. B. (1997). Some thoughts on "normalization" in speech perception. In K. Johnson \& J. W. Mullennix (Eds.), Talker variability in speech processing (pp. 9-32). San Diego, CA: Academic Press.

Rash, F. (2002). The German-Romance language borders in Switzerland. Journal of Multilingual and Multicultural Development, 23, 112-136.

Rittaud-Hutinet, C. (2001). Les français de Besançon: Systèmes phonologiques et énonciation [Varieties of French spoken in Besançon: Phonological systems and pronunciation]. In M.-A. Hintze, T. Pooley, \& A. Judge (Eds.), French accents: Phonological and sociolinguistic perspectives (pp. 97-127). London: National Centre for LanguagesAssociation for French Language Studies.

Schmid, P. M., \& Yeni-Komshian, G. H. (1999). The effects of speaker accent and target predictability on perception of mispronunciations. Journal of Speech, Language, and Hearing Research, 42, 56-64.

Schneider, W., Eschman, A., \& Zuccolotto, A. (2002). E-Prime User's Guide [Computer software manual]. Pittsburgh, PA: Psychology Software Tools.

Sebastián-Gallés, N., Dupoux, E., Costa, A., \& Mehler, J. (2000). Adaptation to time-compressed speech: Phonological determinants. Perception \& Psychophysics, 62, 834-842.

Sebastián-Gallés, N., Dupoux, E., Segui, J., \& Mehler, J. (1992). Contrasting syllabic effects in Catalan and Spanish. Journal of Memory and Language, 31, 18-32.

Singy, P. (Ed). (2002). Le Français Parlé dans le Domaine Francoprovençal: une Réalité Plurinationale [Spoken French in the Francoprovençal domain: A multinational reality]. Bern, Switzerland: Peter Lang.

Singy, P. (Ed.). (2004). Identités de genre, identités de classe et insécurité linguistique en Suisse Romande [Gender identity, social class identity and linguistic insecurity in French-speaking Switzerland]. Bern, Switzerland: Peter Lang.

Tranel, B. (1987). The sounds of French: An introduction. Cambridge, United Kingdom: Cambridge University Press.

van Wijngaarden, S. J. (2001). Intelligibility of native and nonnative Dutch speech. Speech Communication, 35, 103-114.

Weil, S. A. (2001). Foreign accented speech: Adaptation and generalization. Unpublished master's thesis, Ohio State University.

Wells, J. C. (1982). Accents of English: Vol. 1. An introduction. Cambridge, United Kingdom: Cambridge University Press.

Wingstedt, M., \& Schulman, R. (1987). Comprehension of foreign accents. In W. Dressler (Ed.), Phonologica 1984: Proceedings of the fifth International Phonology Meeting (pp. 339-345). Cambridge, United Kingdom: Cambridge University Press. 
Appendix

List of Stimuli

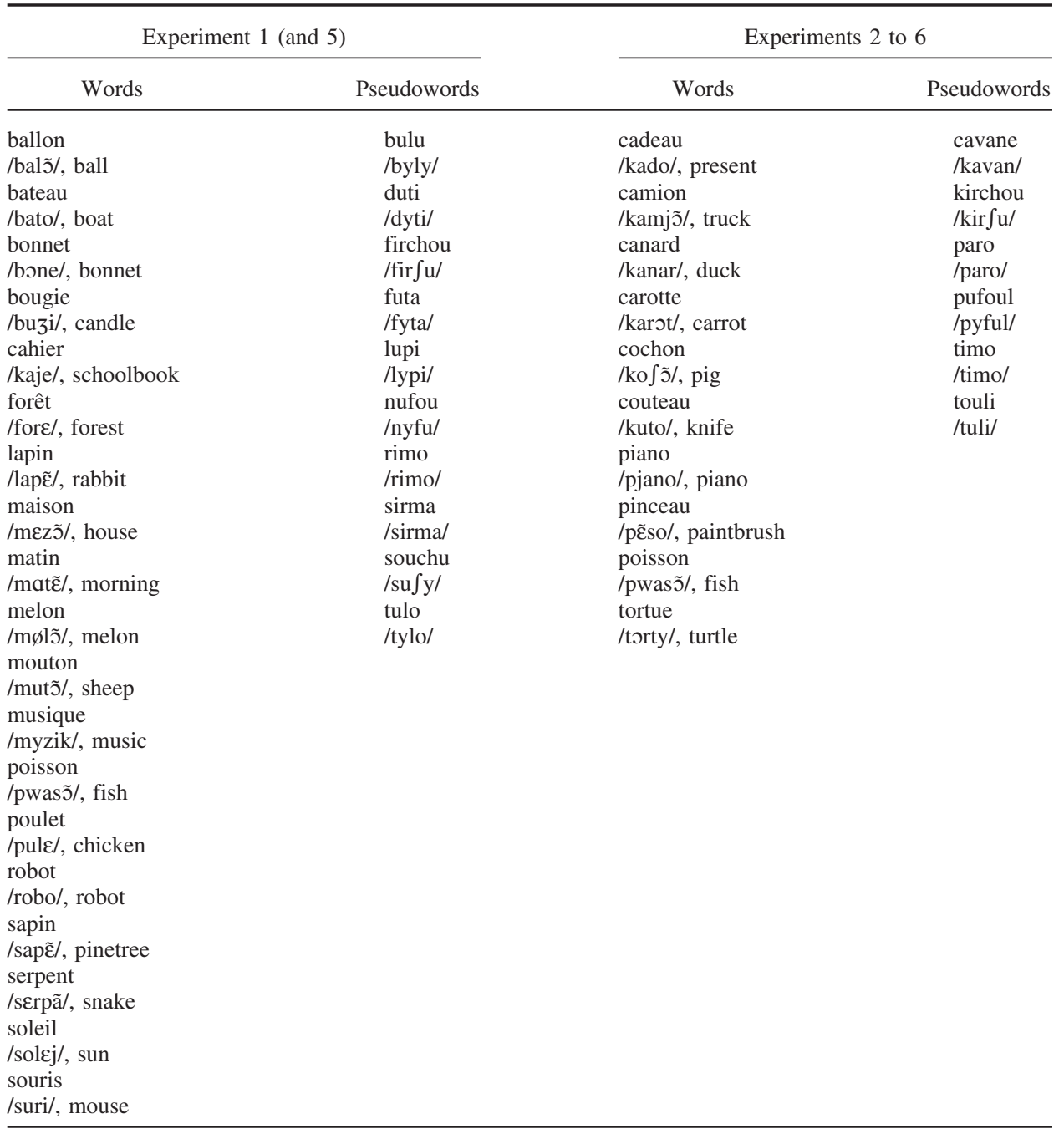

Received April 27, 2005

Revision received May 24, 2006

Accepted May 26, 2006 